\title{
FORAMINÍFEROS PLANCTÔNICOS E BENTÔNICOS CRETÁCEOS DA ILHA DE QUIEPE, FORMAÇÃO ALGODÕES, BACIA DE CAMAMU, BAHIA, BRASIL
}

\author{
GRACE BATISTA CARNEIRO MASCARENHAS \\ Programa de Pós-Graduação em Geologia, UFBA, Campus Universitário de Ondina, Rua Barão de Jeremoabo, s/n., \\ 40170-115, Salvador, BA, Brasil.gracemascarenhas@yahoo.com.br

\section{DENIZE SANTOS COSTA} \\ Bioestratigrafia e Paleoecologia Aplicada (Foraminíferos) Petrobras/CENPES/PDEP/BPA, Rua Horácio Macedo, 950, \\ Cidade Universitária, Ilha do Fundão, Prédio 32, $1^{\circ}$ andar, 21941-915, Rio de Janeiro, RJ, Brasil.denizesc@petrobras.com.br

\section{ENELISE KATIA PIOVESAN} \\ Departamento de Geologia, UFPE, Cidade Universitária, Av. Professor Morais Rego, 1235, 50670-901, \\ Recife, PE, Brasil.katiapiovesan@gmail.com
}

ALTAIR DE JESUS MACHADO

Programa de Pós-Graduação em Geologia, UFBA, Campus Universitário de Ondina, Rua Barão de Jeremoabo, s/n., 40170-115, Salvador, BA, Brasil. altair@ufba.br

\begin{abstract}
CRETACEOUS PLANKTONIC AND BENTHONIC FORAMINIFERA FROM QUIEPE ISLAND, ALGODÕES FORMATION, CAMAMU BASIN, BAHIA, BRAZIL. A sampling program from Camamu Bay and its neighborhood were undertaken to microfossils appraisal of the Algodões Formation outcrops, Camamu Basin. Among the basins of the Brazilian eastern margin, the Camamu Basin is the least known. This study aimed to identify planktonic and benthonic foraminifera of the Algodões Formation, Camamu Basin, and to make biostratigraphic, paleoecological and paleobiogeographic inferences based on the microfauna. The sampling points were located at the Boipeba Island (Boipeba and Tassimirim beaches), and at the Camamu Bay (Barra Grande and Taipús de Fora beaches; Quiepe and Cangaíba islands). In total, 50 rock samples were collected, constituted in great part of calcarenites, besides dolomites, calcilutites and calcareous shale. All the material was subjected to a three laboratory techniques, applied to recover or observe carbonate microfossils: thin section, acetolysis and hydrogen peroxide methods. Only five samples from Quiepe Island showed results. Planktonic foraminifera, belonging to the genus Whiteinella (W. aff. aprica, W. archaeocretacea, $W$. baltica and Whiteinella sp. 1) were identified, besides scarce specimens assigned to Hedbergella (Hedbergella ? sp.1). The paleoecological studies, based on the benthonic foraminifera, revealed predominance of calcareous specimens (Bolivinidae ?), followed by agglutinated taxa (Spiroplectamminidae? and Textulariidae ?). The occurrence of $W$. aff. aprica, W. archaeocretacea and $W$. baltica in the planktonic assemblage suggests a upper Cenomanian to upper Turonian age for the studied section of the Algodões Formation. However, the correlation with ammonites data (Mammites, Kamerunoceras, Romaniceras and Neoptychites) allows to a better biostratigraphic resolution, indicating strata of early-middle Turonian age. The benthonic foraminifera observed (Bolivinidae?, Spiroplectamminidae? and Textulariidae?) indicated the predominance of infaunal organisms that lived on substrates constituted by calcareous mud preferably, and probably occupied the middle-deep neritic paleobathymetric range. The finding of Whiteinella (W. aff. aprica, W. archaeocretacea, $W$. baltica, Whiteinella sp. 1), besides the ostracod specimens of the Sapucariella ex gr. sapucariensis are important biogeographical records in the upper Cretaceous of South America and Africa.
\end{abstract}

Key words: Camamu Basin, Turonian, foraminifera, taxonomy.

RESUMO - Um programa de coleta de amostras na Baía de Camamu e seus arredores foi empreendida para avaliação microfossilífera de afloramentos da Formação Algodões, Bacia de Camamu. Das bacias da margem leste brasileira, a Bacia de Camamu é a menos conhecida. Este trabalho objetivou identificar os foraminíferos planctônicos e bentônicos da Formação Algodões, Bacia de Camamu, bem como realizar inferências bioestratigráficas, paleoecológicas e paleobiogeográficas com base na associação recuperada. As áreas de coleta de amostras foram a Ilha de Boipeba (praias de Boipeba e Tassimirim) e a Baía de Camamu (praias de Barra Grande e Taipús; e as ilhas de Quiepe e Cangaíba). No total, foram coletadas 50 amostras de rochas, constituídas, em sua maioria, de calcarenitos, além de dolomitos, calcilutitos e folhelhos calcíferos. Todo o material foi submetido a técnicas para recuperação ou observação de microfósseis em rochas calcárias: lâmina delgada, métodos de preparação de acetólise e de peróxido de hidrogênio. Somente cinco amostras provenientes da Ilha de Quiepe mostraram 
resultados. Os táxons de foraminíferos planctônicos pertencentes ao gênero Whiteinella ( $W$. aff. aprica, W. archaeocretacea, W. baltica e Whiteinella sp. 1) foram identificados, além de dois espécimes do gênero Hedbergella (Hedbergella? sp.1). Os estudos paleoecológicos, baseados nos foraminíferos bentônicos, revelaram predominância de formas calcárias (Bolivinidae?), seguidas pelas aglutinantes (Spiroplectamminidae? e Textulariidae?). A ocorrência de W. aff. aprica, W. archaeocretacea e W. baltica, na associação planctônica sugeriu idade neocenomaniana a neoturoniana (parte inferior) para a seção estudada da Formação Algodões. Porém, a integração com dados de amonoides (Mammites, Kamerunoceras, Romaniceras e Neoptychites) permitiu uma resolução bioestratigráfica melhor, indicando estratos de idade eo-mesoturoniana. Os foraminíferos bentônicos observados (Bolivinidae?, Spiroplectamminidae? e Textulariidae?) apontou para a predominância de morfogrupos de modo de vida infaunal, que viveram em substratos formados preferencialmente por lamas calcárias, e, que ocuparam provavelmente biótopos situados entre o nerítico médio e nerítico profundo. A observação da associação de espécies do gênero Whiteinella (W. aff. W. aprica, W. archaeocretacea, W. baltica e Whiteinella sp. 1) junto com a espécie de ostracode Sapucariella ex gr. sapucariensis se constituem em registros importantes para a proposição de bioprovíncias no Cretáceo superior das bacias atlânticas da América do Sul e África.

Palavras-chave: Bacia de Camamu, Turoniano, foraminíferos, taxonomia.

\section{INTRODUÇÃO}

Os foraminíferos constituem-se numa ferramenta de grande importância em estudos bioestratigráficos, paleoecológicos e paleobiogeográficos, sendo tema de muitos trabalhos desenvolvidos nas bacias sedimentares do Cretáceo brasileiro (e.g. Koutsoukos, 1989, 1992; Koutsoukos \& Hart, 1990; Silva, 2007, 2011). Estudos aplicando os foraminíferos como indicadores bioestratigráficos, paleoambientais e paleoceanográficos foram realizados na Bacia de Sergipe, compreendendo uma revisão taxonômica dos foraminíferos planctônicos e bentônicos do intervalo Aptiano superiorMaastrichtiano (Koutsoukos, 1989). A revisão se estendeu para o zoneamento bioestratigráfico da bacia, obtido a partir dos foraminíferos planctônicos, onde também foram abordados aspectos paleoecológicos e reconhecidas associações e morfogrupos. Esses dados foram integrados às particularidades sedimentológicas da bacia, tornando possível a proposição de modelos paleoambientais para a área estudada (Koutsoukos, 1989, 1992; Koutsoukos \& Hart, 1990).

Posteriormente, Koutsoukos \& Bengtson (1993) realizaram um trabalho bioestratigráfico integrado para a Bacia de Sergipe, usando como objeto de estudo as associações de foraminíferos e amonoides, com os quais definiram um conjunto de biozonas de idade aptiana a maastrichtiana. Dentre as biozonas de foraminíferos planctônicos, definida nos estudos de Koutsoukos (1989) e Koutsoukos \& Bengtson (1993), ressaltam-se as biozonas abaixo mencionadas, pelas suas correlações com o intervalo do presente estudo desenvolvido na Bacia de Camamu. São elas: Hedbergella (Whiteinella) baltica-Hedbergella $(W$.) brittonensis (Cenomaniano médio-superior); Hedbergella (Whiteinella) aprica-Globigerinelloides bentonensis (Cenomaniano superior), Hedbergella (Whiteinella) archaeocretaceaHeterohelix reussi (Cenomaniano superior-Turoniano inferior) e Hedbergella (Whiteinella) aprica-Hedbergella (Whiteinella) baltica (Turoniano inferior a médio).

Nas bacias sedimentares brasileiras, os foraminíferos ocorrem frequentemente nas seções marinhas desde o Aptiano e sua aplicação em estudos bioestratigráficos e paleoecológicos no Brasil iniciou-se com datações de seções de poços de petróleo e comparação destes com os estratos aflorantes (Schaller, 1969; Noguti \& Santos 1972; Koutsoukos, 1989, 1992).

Correspondendo a um dos trabalhos pioneiros sobre a estratigrafia das bacias de Sergipe e Alagoas, o trabalho de Schaller (1969) mostrou sua importância pela revisão estratigráfica proposta de acordo com as normas técnicas vigentes na época (Códigos de Nomenclatura Estratigráfica Americana e Internacional). Ele propôs novas divisões e definições lito-, bio- e cronoestratigráficas. O estudo foi fundamentado em dados de microfauna (ostracodes e foraminíferos), além de macrofauna (amonoides) e microflora (palinomorfos). Posteriormente, Noguti \& Santos (1972) instituíram um zoneamento preliminar para a plataforma continental brasileira, com base nos foraminíferos planctônicos. Para seção cretácea, foram definidas cinco biozonas, e, particularmente para o intervalo de idade neocenomaniana-santoniana, foi estabelecida a Zona Hedbergella delrioensis.

Silva (2007) realizou uma análise detalhada da bioestratigrafia dos depósitos sedimentares do Cretáceo Superior (Campaniano-Maastrichtiano) da Bacia Pará-Maranhão, estudando radiolários e foraminíferos (planctônicos e bentônicos). A esse estudo também é creditada a incorporação de eventos paleoambientais e paleoecológicos relacionados a essa bacia. Posteriormente, Silva (2011) realizou estudos bioestratigráficos e paleoecológicos com os foraminíferos planctônicos e bentônicos da Bacia de Barreirinhas, localizada na plataforma continental do Maranhão, Nordeste do Brasil. A autora estabeleceu sete biozonas com base em foraminíferos planctônicos (Albiano-Cenomaniano) e detectou eventos paleoecológicos caracterizados por variações climáticas ocorridas neste intervalo.

Estudos taxonômicos, bioestratigráficos e paleoecológicos com foraminíferos, fora do Brasil, também registraram uma microfauna semelhante às encontradas nas bacias do Nordeste brasileiro. Leckie (1985) realizou estudos bioestratigráficos e paleoecológicos com foraminíferos planctônicos e bentônicos da Formação Greenhom, Colorado (EUA). Neste estudo, o autor registrou espécies de foraminíferos planctônicos do Cenomaniano-Turoniano dos gêneros Hedbergella (Pessagno, 1967) e Whiteinella (Brönnimann \& Brown, 1958). Este último gênero representado por $W$. 
aprica, W. archaeocretacea, W. baltica e W. brittonensis. Estudos taxonômicos, paleoambientais e bioestratigráficos, com base em foraminíferos, também foram desenvolvidos em bacias costeiras da África. Dupont (1996) realizou um trabalho taxonômico e bioestratigráfico com as principais espécies de foraminíferos planctônicos do Cretáceo (AptianoCampaniano) da Bacia do Gabão, descrevendo as espécies de $W$. aprica, $W$. archaeocretacea, $W$. baltica dentre outras, definiu 16 biozonas, dentre estas a biozona $W$. aprica-W. archaeocretacea (Cenomaniano superior-Turoniano médio). Gebhardt (1997) documentou os foraminíferos da transição Cenomaniano-Turoniano recuperados em um afloramento localizado no nordeste da Nigéria e identificou espécies de foraminíferos planctônicos, principalmente do gênero Hedbergella. Com base nas associações observadas, o autor contribuiu com informações relacionadas às variações paleoambientais (oxigenação na água) ocorridas no período de deposição dos sedimentos na mesma bacia. Também em seções aflorantes na Nigéria, de idade neocenomaniana-turoniana, Igwe et al. (2015) registraram foraminíferos planctônicos dos gêneros Hedbergella e Whiteinella, incluindo a espécie W. baltica, realizando considerações paleoambientais com base nas associações encontradas.

Para a Formação Algodões na Bacia de Camamu, existem registros de fósseis invertebrados (corais, moluscos e equinoides) em Souza-Lima et al. (2001a, b) e Souza-Lima (2003a, b). Manso (2003) realizou estudos paleoecológicos e paleogeográficos com os equinoides (Aptiano-Albiano) das bacias de Sergipe, Araripe e Camamu (Formação Algodões). Manso \& Souza-Lima (2007) descreveram uma espécie nova de equinoide: Holosalenia bahiensis Manso \& Souza-Lima, 2007, de idade albiana para a Ilha de Cangaíba, mais precisamente no Membro Germânia, da Formação Algodões. Andrade et al. (2004) realizaram um estudo com o gênero Neithea Drouet, 1824, um molusco bivalve, com enfoques estratigráfico, paleobiogeográfico e paleoecológico. Posteriormente, Andrade et al. (2006) descreveram os amonoides de idade turoniana da Ilha de Quiepe, classificados como pertencentes aos gêneros Mammites Laube \& Bruder, 1887; Kamerunoceras Reyment, 1954; Romaniceras Spath, 1923 e Neoptychites Kossmat, 1895.

Com relação aos microfósseis, até o momento, só há o trabalho de Mascarenhas et al. (2016), resultante dos estudos desenvolvidos na tese de doutorado da primeira autora, no qual é documentado a taxonomia dos ostracodes da bacia, bem como considerações paleobiogeográficas e paleoecológicas dos táxons registrados nos afloramentos da Ilha de Quiepe, Formação Algodões, Bacia de Camamu, registrando a espécie Sapucariella ex gr. sapucariensis Puckett et al., 2016 (espécie anteriormente denominada Brachycythere sapucariensis Krömmelbein, 1964, relocada para o novo gênero Sapucariella Puckett et al., 2016), a qual possui grande importância paleobiogeográfica, especialmente no reconhecimento da similaridade faunística entre a América do Sul e a África e na proposição de bioprovíncias no Neocretáceo.

Não há nenhum estudo desenvolvido na Bacia de Camamu com foraminíferos, e, portanto, este trabalho representa o primeiro estudo taxonômico dos foraminíferos planctônicos e bentônicos em depósitos marinhos cretáceos da Formação Algodões. Na presente pesquisa, realizou-se o estudo taxonômico desses foraminíferos, recuperados de uma campanha de amostragem na Baía de Camamu e arredores, além de tecer considerações bioestratigráficas, paleoecológicas e paleobiogeográficas com base nas associações microfossilíferas observadas nos afloramentos da Formação Algodões, na Ilha de Quiepe.

\section{ÁREA DE ESTUDO E CONTEXTO GEOLÓGICO}

Os estudos foram desenvolvidos na Bacia de Camamu, em depósitos do Membro Quiepe, o qual faz parte da Formação Algodões na Ilha de Quiepe. Esta bacia localiza-se na zona costeira do Estado da Bahia, região Nordeste do Brasil, entre os paralelos $13^{\circ}$ e $14^{\circ} \mathrm{Sul}$, abrangendo uma área de 12.000 $\mathrm{km}^{2}$ (Figura 1A). Seu limite norte se dá através da falha de Itapuã, com a Sub-bacia de Jacuípe, e através da falha da Barra e da zona de acomodação de Jaguaripe, com a Bacia do Recôncavo. Seu limite sul é o Alto de Itacaré, que separa as bacias de Camamu e de Almada (Souza-Lima et al., 2001b; Souza-Lima, 2003a; Caixeta et al., 2007).

A Bacia de Camamu originou-se a partir do rifte inicial do processo de separação da América do Sul e a África, no limite norte da região. O rifte, que vinha sendo propagado de sul para norte, bifurcou-se, desenvolvendo as bacias do RecôncavoTucano-Jatobá e permaneceu ativo até o Eoaptiano. Entretanto somente quando o rifte do sistema Sergipe-Alagoas foi estabelecido, é que houve a ruptura entre os dois continentes e a implantação do Oceano Atlântico Sul. A seção marinha da Bacia de Camamu está representada pelas formações Taipus-Mirim, Algodões, Urucutuca, Caravelas e Rio Doce com deposição iniciada no Neoaptiano (Caixeta et al., 2007).

A Formação Algodões possui afloramentos expostos na faixa litoral das ilhas de Tinharé, Boipeba, Tatu, Germânia, Quiepe, Cangaíba, Ilha Grande de Camamu e em diversos pontos da Península de Maraú (Manso \& Souza-Lima, 2007). A Formação Algodões (Figura 2) é representada por um sistema deposicional formado por uma plataforma carbonática rasa, com deposição de calcarenitos e calcilutitos, que gradam para margas e folhelhos na direção da bacia, e está subdividida em dois membros, Germânia e Quiepe. O Membro Germânia é constituído principalmente por calcarenitos e calcirruditos oolíticos/bioclásticos, parcialmente dolomitizados e/ou silicificados, depositados em uma plataforma carbonática rasa. O Membro Quiepe é constituído predominantemente por calcilutitos e margas (Netto et al., 1994; Souza-Lima et al., 2001b; Souza-Lima, 2003a; Caixeta et al., 2007).

O Membro Germânia (afloramentos Ilha de Cangaíba) possui idade neoalbiana, segundo registros dos equinoides Tetragramma malbosi Agassiz \& Desor, 1847 (Manso, 2003) e Holosalenia bahiensis Manso \& Souza-Lima, 2007 (Manso \& Souza-Lima, 2007), do amonoide do gênero Yeharaites Matsumoto, 1991 (Souza-Lima, 2003a) e do molusco bivalve Neithea (Neithea) alpina d'Orbigny, 1847 (Andrade et al., 2004). O Membro Quiepe tem sua idade eo-mesoturoniana 

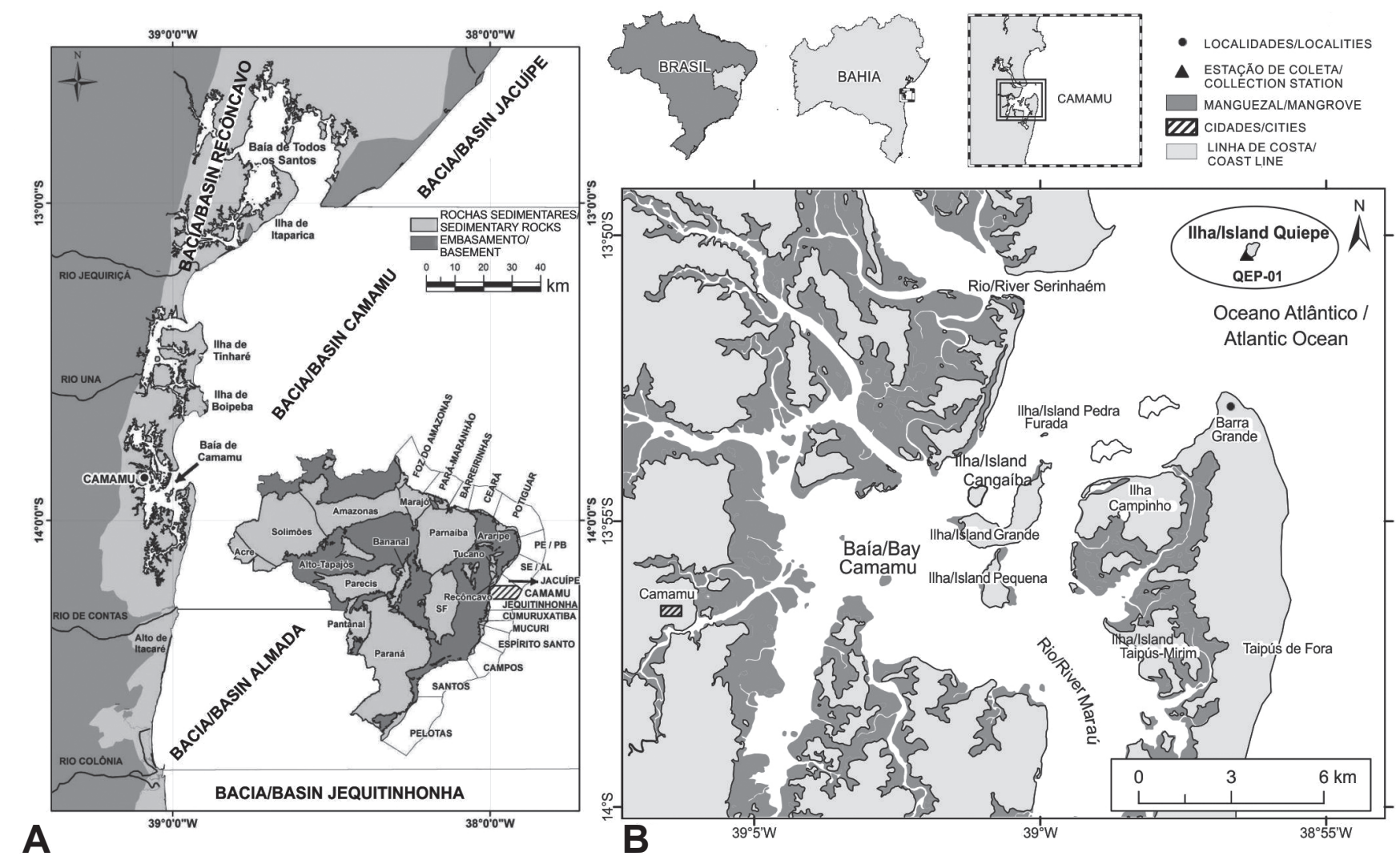

Figura 1. A, mapa de localização da Bacia de Camamu. B, mapa de localização dos afloramentos estudados, em destaque a llha de Quiepe, Baía de Camamu, Bahia, Brasil.

Figure 1. A, location map of the Camamu Basin. B, location map of the studied outcrops in Quiepe Island, Camamu Bay, Bahia, Brazil.

confirmada pelos amonoides encontrados em afloramentos na Ilha de Quiepe, representados pelos gêneros Mammites Laube \& Bruder, 1887, Kamerunoceras Reyment, 1954, Romaniceras Spath, 1923 e Neoptychites Kossmat, 1895 (Andrade et al., 2006).

\section{MATERIAL E MÉTODOS}

O material estudado é proveniente de uma campanha de amostragem de rochas realizada na Baía de Camamu e seus arredores. Porém, somente nas amostras (10) coletadas na Ilha de Quiepe (Figura 1B), a recuperação de espécimes de foraminíferos foi mais significativa. Atualmente, todo esse material está depositado na coleção de fósseis do Museu de Geociências da Universidade Federal da Bahia (UFBA).

$\mathrm{Na}$ Ilha de Quiepe, os espécimes de foraminíferos foram recuperados na porção emersa dos afloramentos da Formação Algodões, litologicamente constituídos, na maioria, por calcarenitos, seguidos de dolomitos, calcilutitos e folhelhos calcíferos.

A descrição dos afloramentos seguiu a metodologia proposta por Bengtson (1983), de modo que foram adotadas terminologias para identificar os afloramentos estudados: Kagq significa Cretáceo, Formação Algodões, Membro Quiepe. A palavra "exposição" indica um afloramento com altura inferior a 0,5 m e "seção" define um afloramento com altura superior a $0,5 \mathrm{~m}$. As abreviaturas entre parênteses referem-se aos códigos dos pontos lançados no mapa (Figura 1B).

Os afloramentos descritos abaixo bordejam a Ilha de Quiepe, só podem ser acessados nos períodos da maré baixa, sendo três os pontos onde foram coletadas amostras (QUI 1, QUI 2 e QUI 3). O acesso a este afloramento se dá pela Baía de Camamu por barco a motor (Figura 3).

Quiepe 1 (QEP-01). UTM 8.470.084N/506.527E. Mapa topográfico folha: SD-24-V-VI-4-SE. Baía de Camamu. Referências à área, de acordo com Andrade et al. (2006); Mascarenhas et al. (2016). Exposições (inferior a 0,5 m) em forma de arrecifes sobre a planície de maré, bordejando toda a ilha (Figura 3), Kagq: calcarenito e folhelhos cinza claro bastante fossilífero com abundância de amonoides de formas e tamanhos variados (Mascarenhas et al., 2016).

A obtenção dos foraminíferos das amostras de rochas coletadas nos afloramentos iniciou-se pelo processo mecânico de trituração das rochas. Todo o material foi submetido a três técnicas de laboratório aplicadas na análise micropaleontológica, principalmente em rochas calcárias: confecção de lâmina delgada e métodos de acetólise e de desagregação da rocha com peróxido de hidrogênio. Contudo, este último método, consultado em Wanderley (2004), foi o que se mostrou mais eficiente na recuperação dos microfósseis. Os espécimes recuperados foram triados com o auxílio de 


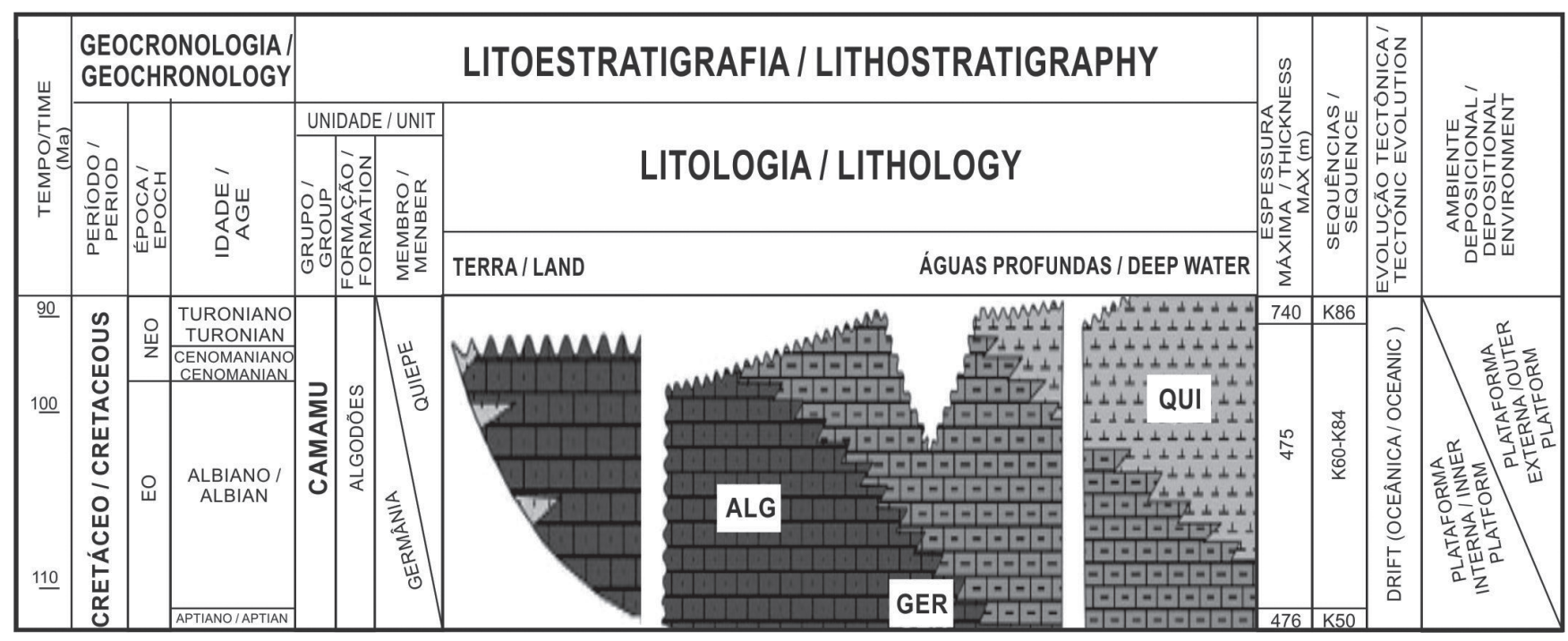

Figura 2. Carta estratigráfica da seção marinha aptiana-turoniana da Formação Algodões, Bacia de Camamu (modificado de Caixeta et al., 2007). Abreviaturas: ALG, Formação Algodões; GER, Membro Germânia; QUI, Membro Quiepe.

Figure 2. Stratigraphic chart of the Aptian-Turonian marine section from the Algodões Formation, Camamu Basin (modified from Caixeta et al., 2007). Abbreviations: ALG, Algodões Formation; GER, Germânia Member; QUI, Quiepe Member.

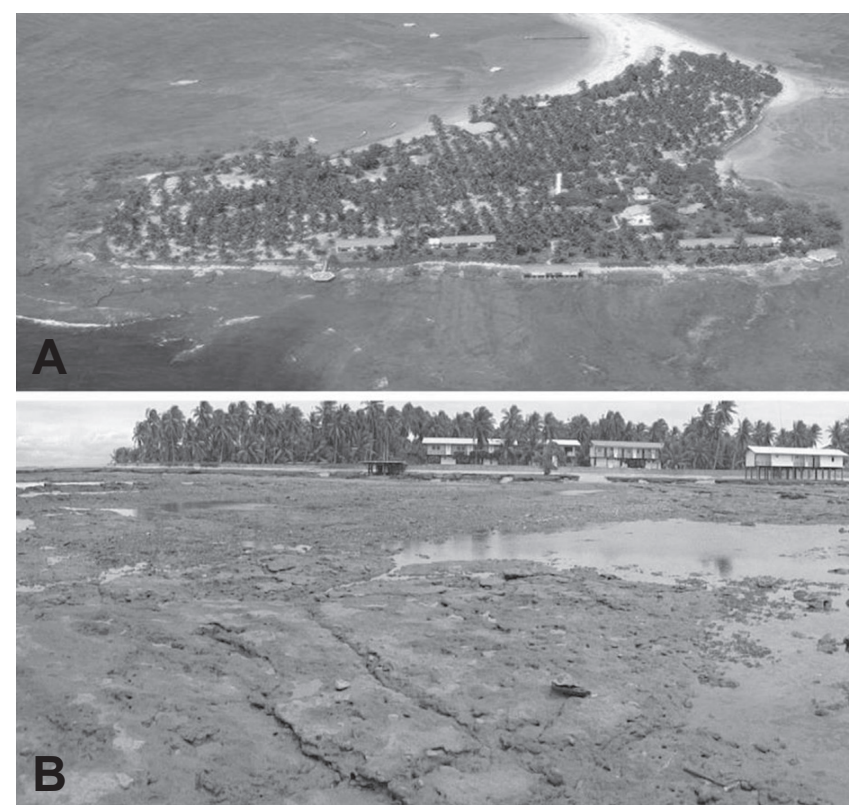

Figura 3. A, vista aérea da Ilha de Quiepe. B, afloramentos expostos em planície de maré, Membro Quiepe, Formação Algodões, Bacia de Camamu, Bahia, Brasil.

Figure 3. A, aerial view of the Quiepe Island B, outcrops exposed in tidal plain, Quiepe Member, Algodões Formation, Camamu Basin, Bahia, Brazil.

estereomicroscópio binocular e, posteriormente, colados em lâminas associativas de Franke para análise detalhada das feições morfológicas. Foram escolhidos exemplares em melhor estado de preservação para realização de fotografias em Microscópio Eletrônico de Varredura (MEV), marca Zeiss e modelo EVO-40, realizadas no Laboratório Petrobras/
CENPES/PDEP/BPA. Após a etapa de análise sistemática dos foraminíferos, procedeu-se com os estudos bioestratigráficos, paleoecológicos e paleobiogeográficos, com base nos dados reunidos anteriormente.

Os resultados bioestratigráficos aqui apresentados tiveram como base o arcabouço para foraminíferos planctônicos do Encontro do Grupo de Trabalho de Microfósseis do Cretáceo Superior, 2011, do programa Times Scale Creator (TSC), versão TSC 6.4, disponível em $<$ https://engineering.purdue. edu/Stratigraphy/tscreator/index/>. O trabalho de referência cronoestratigráfica utilizado no presente estudo foi Gradstein et al. (2012).

A atribuição supragenérica adotada neste trabalho para o estudo taxonômico dos foraminíferos planctônicos segue Loeblich \& Tappan (1988), com exceção do supergrupo (Cavalier-Smith, 2002) e da classe (D’Orbigny, 1826). Para as categorias genérica e específica, adotou-se o catálogo "Mesozoic Planktonic Foraminiferal Taxonomic Dictionary" $<$ http://portal.chronos.org $>$, além da consulta de bibliografia especializada (Robaszynski \& Caron, 1979; Caron, 1985, Premoli-Silva \& Verga, 2004). Para os foraminíferos bentônicos, as atribuições supragenéricas estão de acordo com Loeblich \& Tappan (1988). Além do trabalho de Loeblich \& Tappan (1988) foram consultados para a análise taxonômica dos foraminíferos bentônicos Koutsoukos (1989) e Bolli et al. (1994).

A análise taxonômica foi complementada pela contagem dos espécimes distribuída pelos táxons identificados. A nomenclatura aberta foi empregada quando não foi possível reconhecer a espécie.

Os espécimes ilustrados foram depositados na coleção de fósseis do Museu de Geociências da Universidade Federal da Bahia (UFBA), sob os números curatoriais: MGUFBA1476, MGUFBA1477, MGUFBA1478, MGUFBA1479, 
MGUFBA1480, MGUFBA1481, MGUFBA1482, MGUFBA1483, MGUFBA1484, MGUFBA1485.

Abreviação: QUI, Membro Quiepe.

\section{SISTEMÁTICA PALEONTOLÓGICA}

\section{Foraminíferos planctônicos}

A análise dos foraminíferos planctônicos atestou que a preservação das testas dos espécimes é ruim por estarem totalmente a quase totalmente dissolvidas, restando por vezes somente o molde interno. Estas características preservacionais impediram uma análise taxonômica acurada. Nas listagens sinonímicas apresentadas foram referenciados preferencialmente trabalhos relacionados às bacias do Atlântico Sul, que apresentam descrições e boas imagens.

Supergrupo RHIZARIA Cavalier-Smith, 2002

Classe FORAMINIFERA d'Orbigny, 1826

Ordem FORAMINIFERIDA Eichwald, 1830

Subordem GLOBIGERININA Delage \& Héroaurd, 1891

Superfamília ROTALIPORACEA Sigal, 1958

Família HEDBERGELLIDAE Loeblich \& Tappan, 1961

Subfamília HEDBERGELLINAE Loeblich \& Tappan, 1961

Hedbergella Brönnimann \& Brown, 1958

Hedbergella? sp. 1

(Figuras 3A1-3)

Material. Duas carapaças, QUI 1 (dois espécimes).

Material figurado. MGUFBA1476.

Distribuição estratigráfica. Cenomaniano superiorTuroniano superior (intervalo estudado).

Características. Carapaça com trocoespira baixa, com cerca de três voltas; 5 câmaras na última volta, aumentando moderadamente de tamanho; suturas radiais, deprimidas nos lados espiral e umbilical; umbílico pequeno e profundo; abertura umbilical-extraumbilical não observada, parede calcária, finamente perfurada.

Whiteinella Pessagno, 1967

Whiteinella aff. W. aprica (Loeblich \& Tappan, 1961)

(Figuras 4B1-3)

1961 Ticinella aprica Loeblich \& Tappan, p. 292, pl. 4, figs. $14-16$.

1970 Whiteinella aprica (Loeblich \& Tappan) Eicher \& Worstell, p. 314, pl. 11, Figura 7a-c.

1979 Whiteinella aprica (Loeblich \& Tappan) Robaszynski \& Caron, v.1, p. 157, 160, pl. 32, figs. 1-2.

1985 Whiteinella aprica (Loeblich \& Tappan) Leckie, p. 146, pl. 1, figs. $1-4$.

1996 Whiteinella aprica (Loeblich \& Tappan) Dupont, p. 109 , pl. 7, figs. 8-9.

1989 Hedbergella (Whiteinella) aprica (Loeblich \& Tappan). Koutsoukos, p. 233, pl. 27, figs. 1-6.
2004 Whiteinella aprica (Loeblich \& Tappan) Premoli-Silva \& Verga, p. 215, pl. 145, figs. 1-2.

Material. 27 carapaças. QUI 1 (15 espécimes), QUI 2 (oito espécimes), QUI 3 (quatro espécimes).

Material figurado. MGUFBA1477.

Distribuição estratigráfica. Zonas Rotalipora cushmani, Whiteinella archaeocretacea, Helvetoglobotruncana helvetica e Marginotruncana schneegansi. (Cenomaniano superiorTuroniano superior). De acordo com Gradstein et al. (2012), o topo da Zona M. schneegansi é posicionado na parte inferior do Turoniano superior.

Características. Carapaça com trocoespira muito baixa com duas voltas; periferia arredondada, contorno periférico lobado; presença de cinco a seis câmaras globulares na última volta, que aumentam gradualmente em tamanho; abertura primária em arco interiomarginal, umbilical a extraumbilical, parede calcária com superfície rugosa.

Observações. Esta espécie difere de Anaticinella, de acordo com Chronos, e de Ticinella multiloculata Morrow, 1934 por apresentar uma quantidade menor de câmaras por volta, por estas câmaras serem globulares em vez de ligeiramente subangulares, e por apresentar a superfície um pouco mais rugosa. Distingue-se de Whiteinella archaeocretacea Pessagno, 1967 por apresentar câmaras mais numerosas, globulares e tamanhos geralmente maiores.

Whiteinella archaeocretacea Pessagno, 1967

(Figuras 4C1-3)

1967 Whiteinella archaeocretacea Pessagno, p. 298-299, pl. 51, figs. 2-4; pl. 54, figs. 19-25; pl. 100, fig. 8.

1979 Whiteinella archaeocretacea (Pessagno) Robaszynski \& Caron, v. 1, p. 161, 167-168, pl. 33, figs. 1-3, pl. 34, figs. 1-2. 1980 Whiteinella archaeocretacea (Pessagno) Wonders, p. 129, pl. 3, figs. 1a-c.

1985 Whiteinella archaeocretacea (Pessagno) Leckie, p. 146, pl. 1, figs. 5-6.

1989 Hedbergella (Whiteinella) archaeocretacea (Pessagno) Koutsoukos, p. 234, pl. 27, figs. 7-12.

1996 Whiteinella archaeocretacea (Pessagno) Dupont, p. 113, pl. 9, figs. 10.

2004 Whiteinella archaeocretacea (Loeblich \& Tappan) Premoli-Silva \& Verga, p. 215-216, pl. 145, figs. 3-4; pl.146, fig. 1.

Material. 20 carapaças. QUI 1 (13 espécimes), QUI 2 (quatro espécimes), QUI 3 (três espécimes).

Material figurado. MGUFBA1478.

Distribuição estratigráfica. Zonas Rotalipora cushmani, Whiteinella archaeocretacea, Helvetoglobotruncana helvetica, Marginotruncana schneegansi, Dicarinella concavata e D. asymetrica (Cenomaniano superiorSantoniano).

Características. Carapaça com trocoespira baixa, lobada, com 4-5 câmaras na última volta; câmaras inicialmente esféricas, tornando-se ovaladas em seção transversal na 


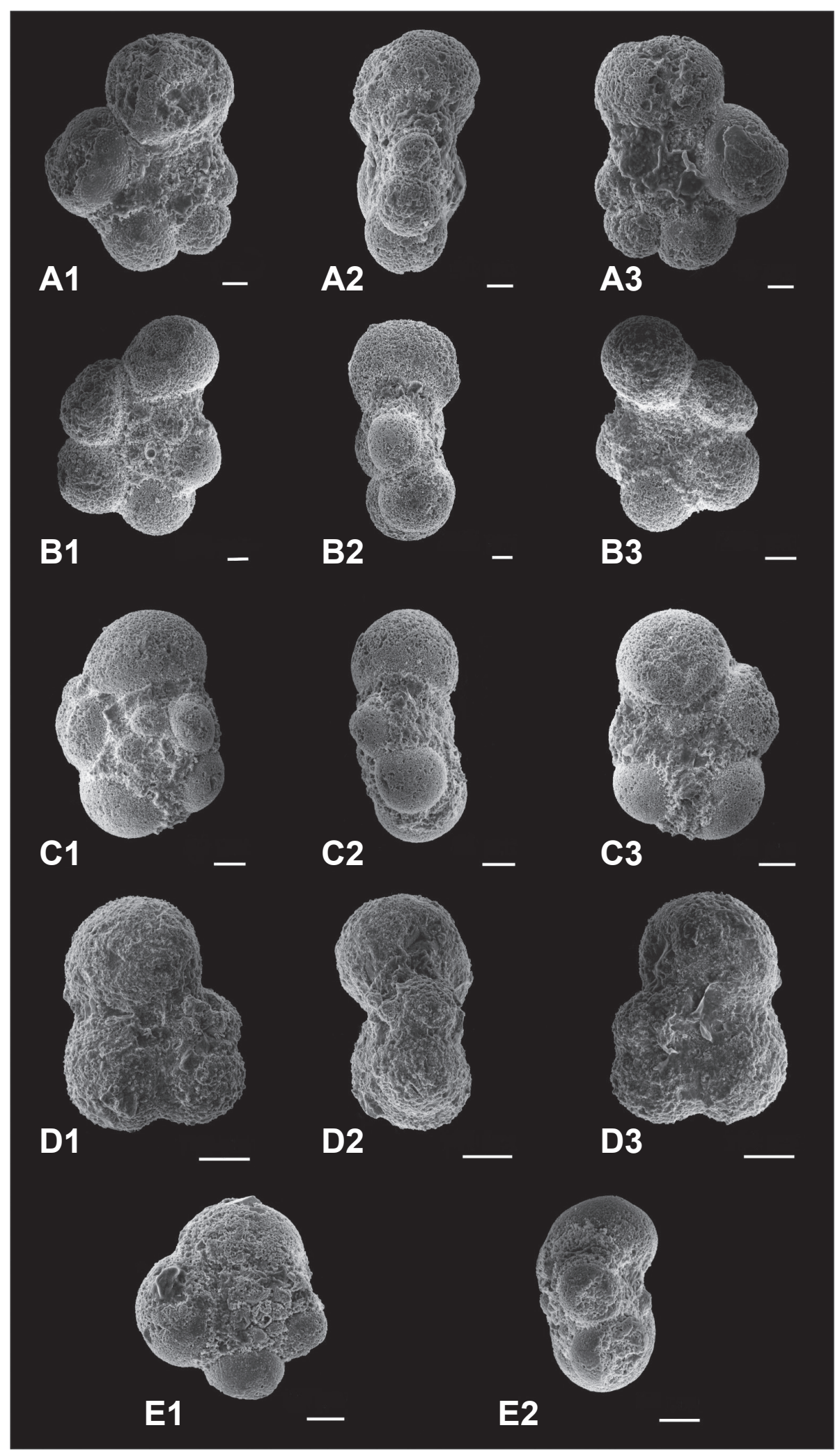

Figura 4. A, Hedbergella? sp. 1 (QUI 1): 1, vista espiral; 2, vista lateral; 3, vista umbilical. B, Whiteinella aff. W. aprica (QUI 1): 1, vista espiral; 2, vista lateral; 3, vista umbilical. C, W. archaeocretacea (QUI 1): 1, vista espiral; 2, vista lateral; 3, vista umbilical. D, W. baltica (QUI 1): 1, vista espiral; 2, vista lateral; 3, vista umbilical. E, Whiteinella sp. 1 (QUI 1): 1, vista espiral; 2, vista lateral. Escalas: A1-A3 = 40 um; B1-B3 = 200 um; C1-C3, E1-E2 = 60 um; D1-D3 = 100 um.

Figure 4. A, Hedbergella? sp. 1 (QUI 1): 1, spiral view; 2, side view; 3, umbilical view. B, Whiteinella aff. W. aprica (QUI 1): 1, spiral view; 2 , side view; 3, umbilical view. C, Whiteinella archaeocretacea (QUI 1): 1, spiral view; 2, side view; 3, umbilical view. D, Whiteinella baltica (QUI 1): 1, spiral view; 2, side view; 3, umbilical view. E, Whiteinella sp. 1 (QUI 1): 1, spiral view; 2, side view. Scale bars: A1-A3 = 40 ym; B1-B3 = 200 um; C1-C3, E1-E2 = 60 um; D1-D3 = 100 um. 
última volta, separadas por suturas radiais, depressivas, retas a curvadas. Carapaça com três voltas de câmaras que aumentam rapidamente em tamanho; câmaras das voltas iniciais pequenas, quando comparadas com as da última volta; abertura primária extraumbilical a quase umbilical, parede perfurada, microgranular; superfície da carapaça rugosa.

Observações. Whiteinella archaeocretacea se assemelha muito à $W$. inornata (Bolli), mas difere desta por ter a periferia arredondada e não periferia angular da última câmara.

\section{Whiteinella baltica Douglas \& Rankin, 1969}

(Figuras 4D1-3)

1969 Whiteinella baltica Douglas \& Rankin, p. 198, figs. $9 \mathrm{a}-\mathrm{c}$.

1978 Whiteinella baltica (Douglas \& Rankin) Caron, pl. 4, figs. $4-5$

1979 Whiteinella baltica (Douglas \& Rankin) Robaszynski \& Caron, v. 1 p. 169, 174, pl. 35, figs. 1-5, pl. 36, figs. 1-2 1985 Whiteinella baltica (Douglas \& Rankin) Caron, p. 79, figs. 7. 1-3

1985 Whiteinella baltica (Douglas \& Rankin) Leckie, p. 146, pl. 2, figs. 4-5, 8, 11 .

1989 Hedbergella (Whiteinella) baltica (Douglas \& Rankin) Koutsoukos, p. 234, pl. 27, figs. 13-19

1996 Whiteinella baltica (Douglas \& Rankin) Dupont, p. 109 , pl. 7, figs. 1-2.

2004 Whiteinella baltica Douglas \& Rankin, 1. Premoli-Silva \& Verga, p. 217, pl. 147, figs. 1-3.

Material. Sete carapaças. QUI 1 (dois espécimes), QUI 2 (três espécimes), QUI 3 (dois espécimes).

Material figurado. MGUFBA1479.

Distribuição estratigráfica. Zonas Rotalipora cushmani, Whiteinella archaeocretacea, Helvetoglobotruncana helvetica, Marginotruncana schneegansi, Dicarinella concavata, D. asymetrica e Globotruncanita elevata (Cenomaniano superior-Campaniano inferior).

Características. Carapaça trocoespiral muito baixa, com quatro a cinco câmaras e subesféricas na última volta, periferia axial arredondada; vista espiral com câmaras iniciais que aumentam rapidamente de tamanho, câmaras finais aumentam gradualmente; suturas espirais e umbilicais radiais, ligeiramente curvadas; umbílico raso e largo; abertura primária extraumbilical a umbilical, parede calcária, perfurada; superfície das câmaras de aspecto ríspido.

Observações. Difere de Whiteinella archaeocretacea por esta apresentar testas maiores com maior número de câmaras, com maior cavidade umbilical e compactadas no plano equatorial (vista lateral).

\section{Whiteinella sp. 1}

(Figuras 4 E1-3)

Material. Nove carapaças. QUI 1 (dois espécimes), QUI 2 (cinco espécimes), QUI 3 (dois espécimes).

Material figurado. MGUFBA1480.
Distribuição estratigráfica. Cenomaniano inferiorSantoniano.

Características. Carapaça lobada, trocoespira baixa, 4 câmaras esféricas na última volta, com aumento rápido de tamanho na última volta; parede calcária; superfície da carapaça pustulosa. Este táxon se assemelha ao parátipo 1 da espécie Whiteinella baltica de Douglas \& Rankin, 1969. Entretanto, foi diferenciada pela morfologia externa menos lobada e pelas últimas câmaras serem mais semicirculares.

\section{Foraminíferos bentônicos}

A análise taxonômica dos foraminíferos bentônicos constatou espécimes quase totalmente a totalmente desprovidos de carapaças (dissolução), restando por vezes somente o molde interno. As feições de sutura são pouco visíveis, possivelmente por efeito de recristalização. As condições de preservação de suas carapaças são piores quando comparadas às dos foraminíferos planctônicos. Por essa razão, a classificação taxonômica limitou-se ao reconhecimento da categoria de família.

Subordem TEXTULARIINA Delage \& Hérouard, 1896 Superfamília SPIROPLECTAMMINACEA Cushman, 1927 Família SPIROPLECTAMMINIDAE Cushman, 1927

Spiroplectamminidae? gen. et sp. indet. 1 (Figuras 5A1-2)

Material. Duas carapaças. QUI 2 (um espécime) QUI 3 (um espécime).

Material figurado. MGUFBA1481.

Características. Carapaça bisseriada, raramente se tornando unisseriada; paredes aglutinadas.

Observações. A família Spiroplectamminidae ocorre do Carbonífero ao Holoceno. O estado ruim de preservação do espécime não permitiu uma classificação nas categorias genérica e específica.

Superfamília TEXTULARIACEA Ehrenberg, 1838

Família TEXTULARIIDAE Ehrenberg, 1838

Textulariidae? gen. et sp. indet. 1

(Figuras 5B1-2)

Material. Quatro carapaças, QUI 3 (quatro espécimes). Material figurado. MGUFBA1482.

Características. Carapaças alongadas, bisseriadas, raramente se tornando unisseriadas; parede aglutinada, composta por partículas calcárias ou siliciclásticas agregadas por cimento secretado orgânico ou calcário.

Subordem ROTALIINA Delage \& Hérouard, 1896

Superfamília BOLIVINACEA Glaessner, 1937 Família BOLIVINIDAE Glaessner, 1937

Bolivinidae? gen. et sp. indet. 1

(Figuras 5C1-2) 


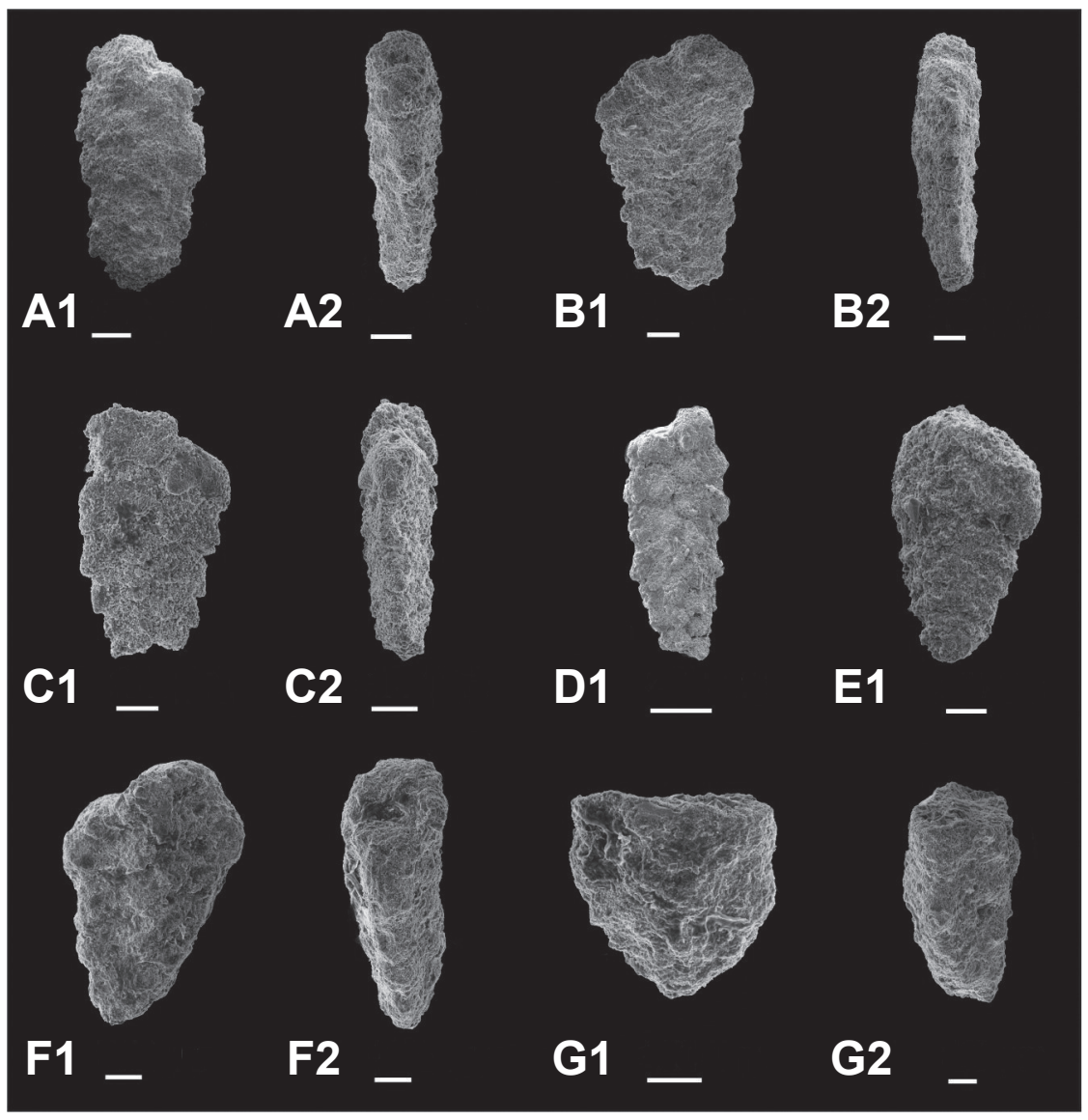

Figura 5. A, Spiroplectamminidae? gen. et sp. indet. 1 (QUI 2): 1, vista lateral; 2, vista lateral. B, Textulariidae? gen. et sp. indet. 1 (QUI 3): 1 , vista lateral; 2, vista lateral. C, Bolivinidae? gen. et sp. indet. 1 (QUI 1): 1, vista lateral; 2, vista lateral. D, Bolivinidae? gen. et sp. indet. 2 (QUI 1): 1, vista lateral. E, Bolivinidae? gen. et sp. indet. 3 (QUI 1): 1, vista lateral. F, Bolivinidae? gen. et sp. indet. 4 (QUI 1): 1, vista lateral; 2 , vista lateral. G, Bolivinidae? gen. et sp. indet. 5 (QUI 1): 1, vista lateral; 2, vista lateral. Escalas: A1-A2, B1-B2, C1-C2, F1-F2, G1 = 100 um; D1 = 200 чm; $\mathrm{E} 1=80$ чm; G2 = 60 чm.

Figure 5. A, Spiroplectamminidae? gen. et sp. indet. 1 (QUI 2): 1, side view; 2, side view. B, Textulariidae? gen. et sp. indet. 1 (QUI 3 ): 1 , side view; 2, side view. C, Bolivinidae? gen. et sp. indet. 1 (QUI 1) 1, side view; $\mathbf{2}$, side view. D, Bolivinidae? gen. et sp. indet. 2 (QUI 1): 1, side view. E, Bolivinidae? gen. et sp. indet. 3 (QUI 1): 1, side view. F, Bolivinidae? gen. et sp. indet. 4 (QUI 1): 1, side view; 2, side view. G, Bolivinidae? gen. et sp. indet. 5 (QUI 1): 1, side view; 2, side view. Scale bars: A1-A2, B1-B2, C1-C2, F1-F2, G1 = 100 um; D1 = 200 ym; E1 = 80 um; G2 = 60 um.

Material. 36 carapaças QUI 1 (seis espécimes), QUI 2 (11 espécimes), QUI 3 (19 espécimes).

Material figurado. MGUFBA1483.

Características. Os espécimes se identificam com a família Bolivinidae pelo seu estágio principal bisserial; câmaras não infladas e que vão ficando mais finas nas extremidades; crescimento gradual de tamanho das câmaras; abertura pouco visível, devido à má preservação dos espécimes.

\section{Bolivinidae? gen. et sp. indet. 2}

(Figura 5D1)

Material. 1 carapaça. QUI 1 (um espécime).

Material figurado. MGUFBA1484.

Características. O espécime se identifica com a família Bolivinidae pelo seu estágio principal bisserial. Entretanto, as câmaras tendem a subglobosas e o crescimento de tamanho das câmaras é lento, mostrando lados quase paralelos; abertura pouco visível, devido à má preservação do espécime.
Bolivinidae? gen. et sp. indet. 3

(Figura 5E1)

Material. Uma carapaça. QUI 1 (um espécime).

Material figurado. MGUFBA1485.

Características. $\mathrm{O}$ espécime se identifica com a família Bolivinidae pelo seu estágio principal bisserial e uma tendência de câmaras mais finas nas extremidades; crescimento gradual de tamanho das câmaras evidenciando morfologia externa triangular; abertura pouco visível, devido à má preservação do espécime.

\section{Bolivinidae? gen. et sp. indet. 4}

(Figuras 5F1-2)

Material. 19 carapaças. QUI 1 (dois espécimes), QUI 2 (quatro espécimes), QUI 3 (13 espécimes).

Material figurado. MGUFBA1486.

Observação. Em função da preservação ruim, parte dos 
espécimes mostra similaridade com a família Fursenkoinidae, pelo arranjo reto das suturas entre as câmaras bisseriadas. Na Figura 4, eles correspondem aos exemplares F1 e E1 (Bolivinidae? gen. et sp. indet. 3)

Bolivinidae? gen. et sp. indet. 5

(Figuras 5G1-2)

Material. Uma carapaça. QUI 1 (um espécime).

Material figurado. MGUFBA1486.

Características. Carapaça bisseriada pelo menos na fase inicial, podendo tornar-se unisseriadas na fase final. Apresentam câmaras estreitas no início que aumentam de tamanho no final da testa, a qual é comprimida, mostrando a periferia subaguda à aguda.

\section{DISCUSSÃO}

\section{Considerações bioestratigráficas}

Estudos bioestratigráficos são importantes, pois fornecem a datação relativa para uma sequência de estratos e permitem a correlação entre seções. Nas análises das amostras QUI 1, QUI 2 e QUI 3 coletadas na Formação Algodões (Ilha de Quiepe), foram contabilizados 65 espécimes de foraminíferos planctônicos, representados pelos gêneros Whiteinella (dominante) e Hedbergella. De acordo com a Tabela 1, a associação planctônica identificada nas amostras acima mencionadas é constituída pelas espécies Whiteinella aff. $W$. aprica, $W$. archaeocretacea, $W$. baltica, Whiteinella sp. 1 e Hedbergella? sp. 1, todas pertencentes à família Hedbergellidae. Mesmo tratando-se de uma associação planctônica pouco diversificada, não apresentando uma boa preservação, e sem a constatação de espécies-guias para a determinação de biozonas, foi possível estabelecer uma datação relativa para os estratos a partir dos bioeventos registrados. Os táxons Whiteinella aff. W. aprica, $W$. archaeocretacea e $W$. baltica foram identificados nas três amostras coletadas na Ilha de Quiepe (QUI 1, QUI 2 e QUI3). Os bioeventos de primeira ocorrência de $W$. archaeocretacea e última ocorrência de Whiteinella aff. $W$. aprica (Figura 6) foram utilizados para inferir o conjunto de biozonas Rotalipora cushmani, W. archaeocretacea, Helvetoglobotruncana helvetica e Marginotruncana schneegansi para essas amostras, conforme o arcabouço bioestratigráfico estabelecido pelo Grupo de Trabalho de Microfósseis do Cretáceo Superior, 2011, Programa Times Scale Creator (TSC), versão TSC 6.4 (Figura 6). Os bioeventos caracterizados sugerem idade neocenomaniana à neoturoniana para o afloramento estudado da Ilha de Quiepe, Formação Algodões, na Bacia de Camamu.

Tabela 1. Distribuição estratigráfica dos táxons de foraminíferos identificados nos afloramentos da llha de Quiepe, Membro Quiepe, Formação Algodões (Cretáceo superior), Bacia de Camamu, Bahia, Brasil.

Table 1. Stratigraphic distribution of foraminifera taxa in outcrops of Quiepe Island, Member Quiepe, Algodões Formation (upper Cretaceous), Camamu Basin, Bahia, Brazil.

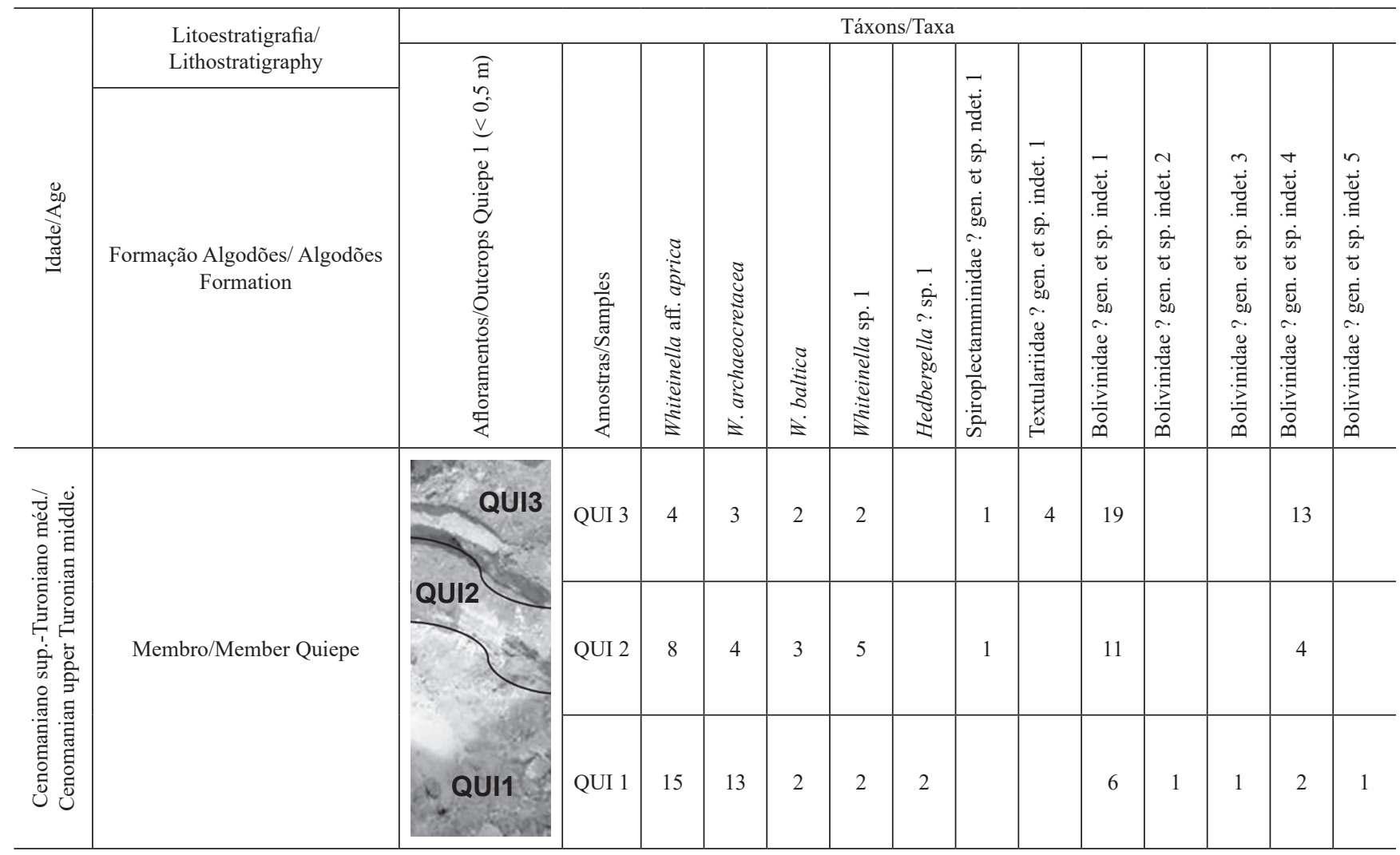




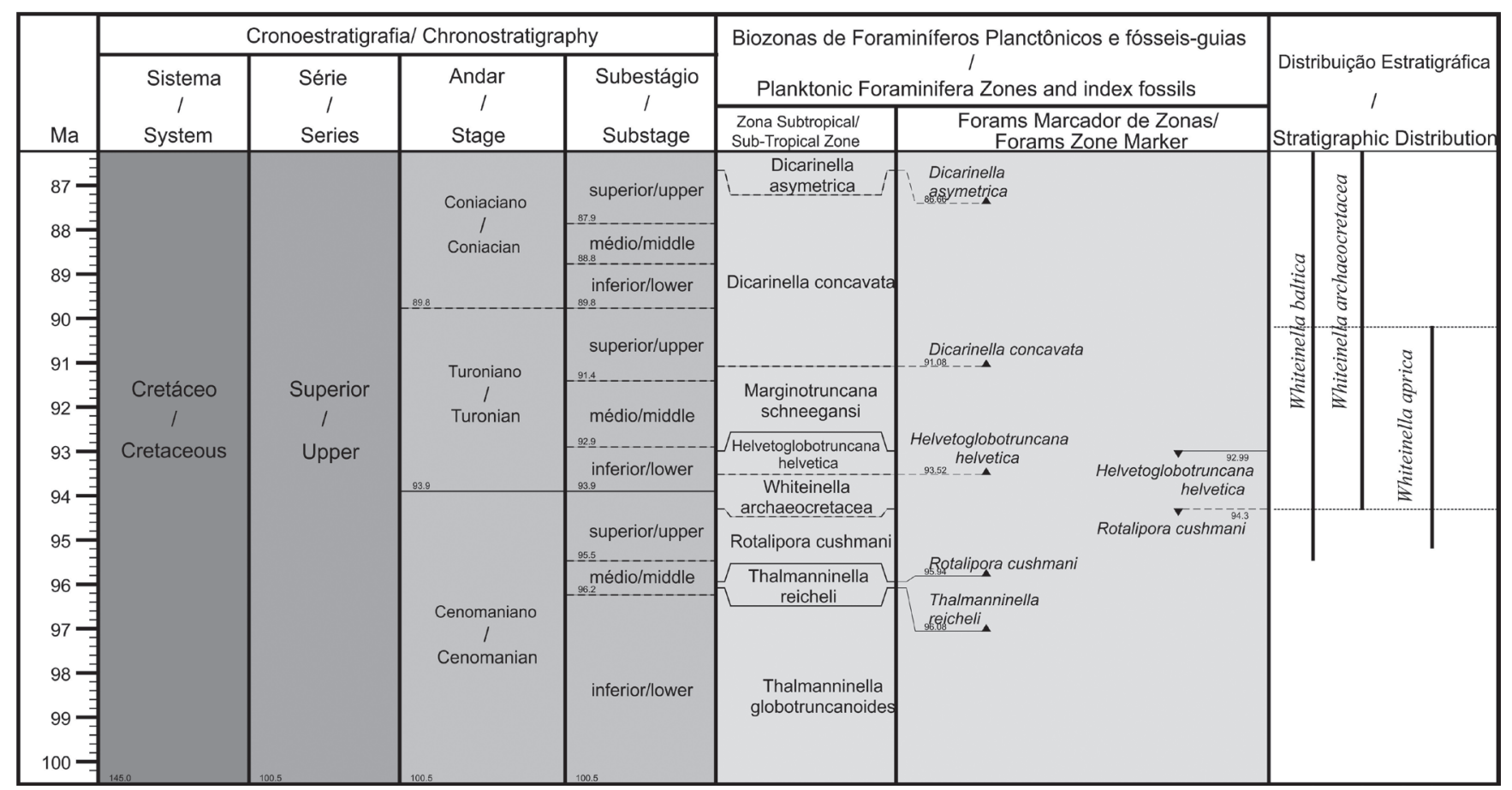

Figura 6. Distribuição estratigráfica das espécies de foraminíferos planctônicos identificadas nas amostras QUI 1, QUI 2 e QUI 3. Arcabouço bioestratigráfico de referência usado no presente estudo de acordo com o Grupo de Trabalho de Microfósseis do Cretáceo Superior (Encontro 2011, Times Scale Creator, versão TSC 6.4).

Figure 6. Stratigraphical distribution chart of the planktonic foraminifera species identified in the QUI 1, QUI 2 and QUI 3 samples. Biostratigraphical framework established to this work, according to The Late Cretaceous Microfossil Working Group (Meeting 2011, TSC, 6.4).

As três espécies de foraminíferos da família Hedbergellidae recuperadas do afloramento da Ilha de Quiepe (Whiteinella aff. W. aprica, W. archaeocretacea e W. baltica), juntamente com os ostracodes Sapucariella ex. gr. sapurariensis que foram registrados nos mesmos pontos de coleta (Mascarenhas et al., 2016), podem ser utilizados para correlacionar bioestratigraficamente intervalos da Bacia de Camamu com seções coevas de outras bacias do Nordeste brasileiro, em especial da Bacia de Sergipe (Tabela 2), e da Bacia do Gabão na África.

Koutsoukos (1992) e Koutsoukos \& Bengtson (1993) definiram biozonas para o Cretáceo superior da Bacia de Sergipe utilizando foraminíferos e amonoides. A distribuição das espécies do gênero Whiteinella (W. aff. W. aprica, $W$. archaeocretacea e $W$. baltica) foi posicionada nos andares Cenomaniano-Turoniano. Para a Bacia do Gabão, África, Dupont (1996) posicionou bioestratigraficamente as espécies de foraminíferos planctônicos $W$. aprica, $W$. archaeocretacea, $W$. baltica no intervalo Cenomaniano superior-Turoniano médio. Nas bacias de Sergipe e Potiguar, Viviers et al. (2000) registraram o ostracode Sapucariella sapucariensis (Turoniano ao Coniaciano). Essa mesma espécie de ostracode ( $S$. ex gr. sapucariensis) também foi registrada na Bacia de Camamu para o Turoniano inferior a médio (Mascarenhas et al., 2016). Estes dados corroboram o trabalho de Andrade et al. (2006), que indicou a idade eomesoturoniana para o Membro Quiepe, Formação Algodões, a partir dos amonoides (Mammites, Kamerunoceras,
Romaniceras e Neoptychites) encontrados nos afloramentos da Ilha de Quiepe.

\section{Considerações paleoecológicas}

Os foraminíferos bentônicos respondem às variações do meio em que vivem, e por isso têm sido muito utilizados tanto em estudos ecológicos (Murray, 2006) quanto paleoecológicos (Koutsoukos \& Hart, 1990). Murray (2006) listou uma série de fatores mensuráveis no controle ambiental atuantes na distribuição dos foraminíferos, classificando-os como fatores abióticos (salinidade, temperatura, oxigênio dissolvido na água, nutrientes, marés e substrato), fatores bióticos (competição, espaço, suprimento alimentar) e fatores bióticos/abióticos (distúrbios, controle de anoxia). Sob o enfoque biológico, sabe-se que as carapaças dos foraminíferos exercem diferentes funções, como, por exemplo, defesa contra predação e flagelos ambientais, equilíbrio na superfície das águas e estratégias alimentares no nicho onde vivem. Tais características mostram sua importância nos mecanismos de sobrevivência e modo de vida, principalmente dos foraminíferos bentônicos, servindo de base para o agrupamento em morfogrupos. Os morfogrupos reúnem aqueles foraminíferos bentônicos cujas carapaças exibem afinidades morfológicas, composicionais e de distribuição paleoambiental, que refletem seu modo de adaptação ao meio (Koutsoukos \& Hart, 1990).

Koutsoukos \& Hart (1990) estudaram as variações morfológicas das carapaças dos foraminíferos bentônicos e as 
Tabela 2. Bioestratigrafia com base em amonoides, foraminíferos planctônicos e ostracodes das bacias cretácicas do Nordeste brasileiro e Gabão, África.

Table 2. Biostratigraphy based on amonoides, planktonic foraminifera and ostracods studies in Cretaceous basins, Brazilian northeastern and Gabon, Africa.

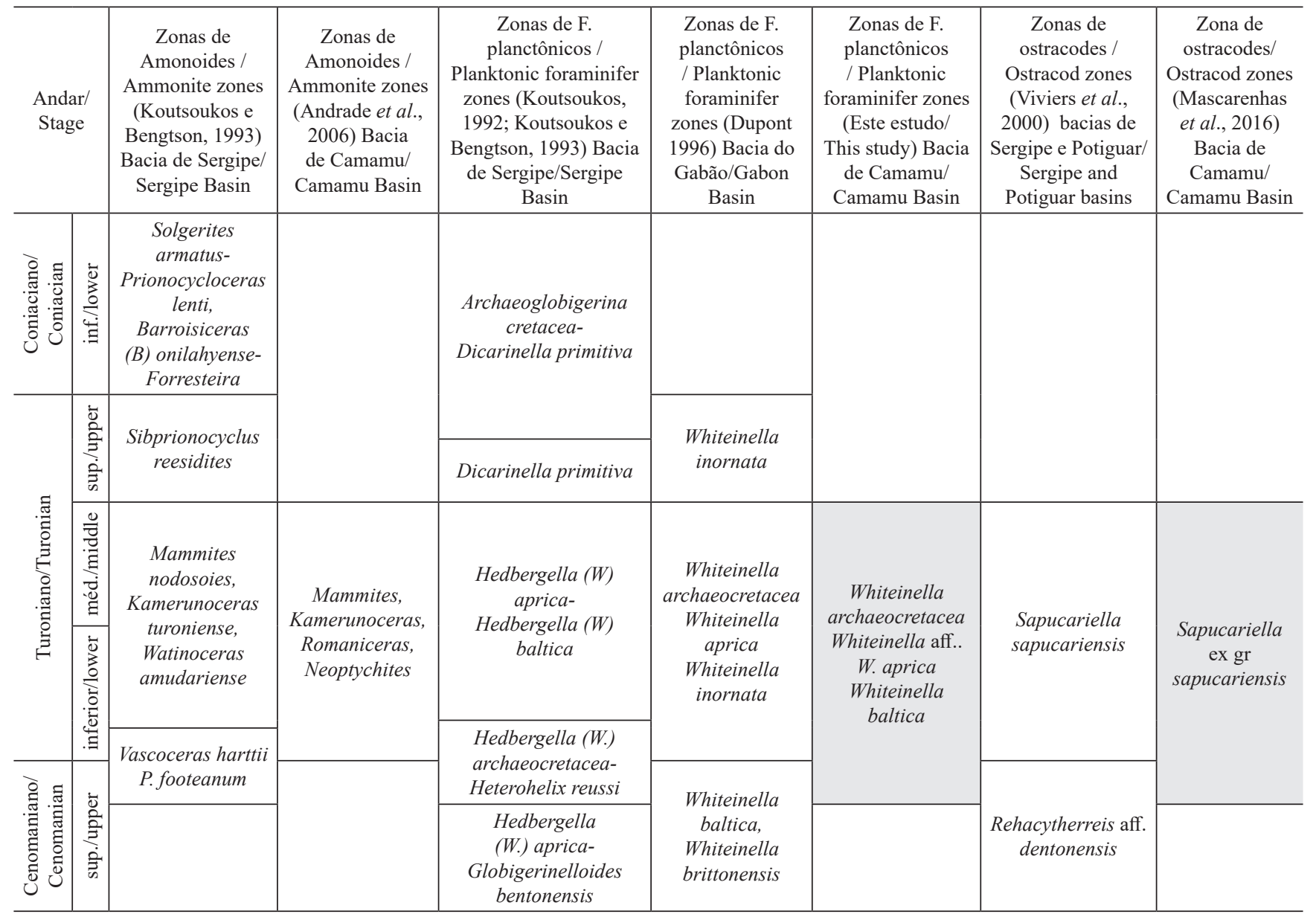

correlacionaram às adaptações comportamentais (condições alimentares e micro-habitat) desses organismos frente às mudanças paleoambientais que se sucederam ao longo do tempo na Bacia de Sergipe. Fatores como estabilidade ambiental, temperatura, concentração de oxigênio dissolvido na água e salinidade estão entre as causas que controlaram a distribuição e evolução das paleocomunidades de foraminíferos bentônicos.

Recentemente, Hart \& Koutsoukos (2015) analisaram associações de morfotipos planctônicos e bentônicos (incluindo os hedbergelídeos, bolivinídeos e textulariídeos) do Golfo do México e bacias das margens passivas do Oceano Atlântico. Nos estudos paleoecológicos e paleoceanográficos realizados por esses autores, grandes eventos de extinção na passagem Cenomaniano-Turoniano e no limite Cretáceo/ Paleógeno foram constatados, relacionando assim associações de foraminíferos a importantes eventos anóxicos e de mudanças do nível do mar e vulcanismo.

As amostras da Ilha de Quiepe foram coletadas em exposições dos arrecifes de planície de maré. Os foraminíferos bentônicos das associações recuperadas nessas amostras variaram tanto na abundância quanto no número de táxons. A amostra QUI 1 apresentou 11 espécimes pertencentes a um táxon. A amostra QUI 2 apresentou 16 espécimes correspondentes a dois táxons. E na amostra QUI 3, foram recuperados 37 espécimes de foraminíferos bentônicos, representativos de três táxons.

Um aspecto observado em todas as amostras foi a preservação precária dos espécimes de foraminíferos bentônicos. As carapaças se mostraram quase totalmente a totalmente dissolvidas, sobrando apenas um molde interno e indícios de recristalização. Este fato impediu a visualização de estruturas diagnósticas, o que limitou a classificação taxonômica dos espécimes ao nível de família.

As principais características das associações de foraminíferos recuperadas nas amostras QUI 1, QUI 2 e QUI 3 seguem descritas abaixo com ênfase na fauna bentônica. As interpretações foram efetuadas com base nos trabalhos de Koutsoukos \& Hart (1990) e Hart \& Koutsoukos (2015).

Nas amostras QUI 1, QUI 2 e QUI 3, além dos foraminíferos (planctônicos e bentônicos) foram observados ostracodes e amonoides (Mascarenhas et al., 2016; Andrade et al., 2006). 
Na amostra QUI 1 a ocorrência de foraminíferos bentônicos é subordinada à dos planctônicos (Whiteinella aff. W. aprica, $W$. archaeocretacea e $W$. baltica), representando apenas $20 \%$ da associação recuperada, ocorrendo exclusivamente espécimes de bolivinídeos (Tabela 1). De acordo com Koutsoukos \& Hart (1990), esta família apresenta morfotipos com testas alongadas, no início com câmaras estreitas, que aumentam de tamanho no final da testa e mostram arranjo bisseriado; a testa é comprimida e com periferia subaguda. Compreendem organismos que se alimentaram de detritos e bactérias (detritívoros) e de hábitos infaunais, cuja morfotipo alongado minimiza o esforço de deslocamento dentro do sedimento à procura de alimento. Viviam preferencialmente em substratos lamosos calcários. Esses morfogrupos de hábitos infaunais poderiam ter ocupado biótopos localizados em dois intervalos paleobatimétricos (Ingle Junior, 1980), nerítico médio a profundo (50-200 m), e, entre o batial superior a médio (200 m-1000 m). Representaram associações móveis de detritívoros, infaunais, que habitaram sedimentos de granulometria fina (lamosa) em locais de fundo relativamente estável e ricos em nutrientes. Segundo, Koutsoukos \& Hart (1990), os bolivinídeos foram comuns em seções de idade cenomaniana-turoniana na Bacia de Sergipe.

A amostra QUI 2 mostra que a relação planctônico/ bentônico da associação recuperada é mais equilibrada. Os bentônicos perfazem $44 \%$ da associação, sendo constituídos por mais de $90 \%$ de formas calcárias (bolivinídeos) e 10\% por formas aglutinantes (spiroplectamminídeos). Conforme já descrito anteriormente, os bolivinídeos mostram testas alongadas, com câmaras estreitas, aumentando de tamanho para o final da carapaça, com arranjo bisseriado e periferia subaguda a aguda. Já os spiroplectamminídeos são morfotipos de testa composta por partículas calcárias ou siliciclásticas agregadas por cimento secretado orgânico ou calcário. Como o morfogrupo anterior, são alongados, com tipo de enrolamento e arranjo das câmaras variados; pode ser de unisserial a multisserial. Ambos os táxons viveram preferencialmente em substratos lamosos calcários, formados por grupos tróficos de detritívoros (decompositores de detritos e bactérias) e de hábito de vida infaunal. A presença e dominância dos bolivinídeos indicam ocupação de biótopos localizados em paleobatimetrias nerítico médio e profundo, e, batial superior e médio (200-1000 m). Entretanto, a ocorrência de espécimes de spiroplectamminídeos pode sugerir biótopos desde paleoambientes parálicos até biótopos localizados em paleobatimetria batial, o que corrobora com os bolivinídeos.

A amostra QUI 3 foi a que apresentou maior número de espécimes (48) e o menor número de foraminíferos planctônicos (11). Os foraminíferos bentônicos dominaram a associação (77\%), constituída por bolivinídeos, textularí́deos e spiroplectamminídeos. De acordo com Koutsoukos \& Hart (1990), os bolivinídeos apresentam morfotipos de carapaças alongadas e planas, com câmaras estreitas, que aumentam de tamanho no final da testa e possuem periferia subaguda a aguda. O predomínio dos bolivinídeos na amostra QUI 3 revelou uma associação de organismos infaunais móveis detritívoros, que habitaram preferencialmente sedimentos lamosos calcários, em locais de relativa estabilidade de fundo e ricos em nutrientes.

Os resultados paleoecológicos mostraram que as associações de foraminíferos bentônicos, registradas nas amostras QUI 1, QUI 2 e QUI 3, assinalam domínio das formas calcárias (bolivinídeos) e a ocorrência secundária de foraminíferos bentônicos aglutinantes (spiroplectamminídeos e textulariídeos), ambos integrantes de morfogrupos de modo de vida infaunal e que teriam habitado sedimentos calcários de granulometria fina (lamosos).

Com base na predominância dos bolivinídeos e de acordo com o modelo de distribuição faunística proposto para o Cenomaniano-Turoniano por Koutsoukos \& Hart (1990), estima-se que para as amostras QUI 1, QUI 2 e QUI 3, do afloramento Quiepe 1, paleobatimetria entre nerítico médio e profundo (50 a $400 \mathrm{~m}$ ).

Junto aos espécimes de foraminíferos bentônicos da Ilha de Quiepe foram registrados espécimes de ostracodes que também contribuíram para a compreensão e reconstrução paleoambiental (Mascarenhas et al., 2016). Os ostracodes pertencem à Família Trachyleberididae, bem representada nos depósitos marinhos das bacias do Nordeste brasileiro, como Sergipe, Potiguar e Pará-Maranhão (Viviers et al., 2000; Piovesan et al., 2009; 2014a, b). Eles são indicativos de um paleoambiente marinho com salinidade normal. Já o gênero de ostracode Matronella, registrado nas amostras QUI 2 e QUI 3 do Membro Quiepe mostra características morfológicas, como a presença de tubérculos oculares bem desenvolvidos, que, segundo Babinot (1995) e Fauth (2002), indicam terem vivido em paleoambientes de águas não muito profundas, restritos à zona fótica.

A associação faunística do afloramento Quiepe 1 (QUI 1, QUI 2 e QUI 3), composta pelos ostracodes da família Trachyleberididae e pelas famílias de foraminíferos bentônicos Bolivinidae, Textularidae e Spiroplectamminidae reforçam a proposição de terem vivido em biótopos de paleobatimetrias entre o nerítico interno, médio a profundo (0-400 m), com salinidade normal, em paleoambiente de plataforma carbonática.

Nas amostras QUI 2 e QUI 3 foram registrados foraminíferos bentônicos spiroplectamminídeos e textulariídeos e ostracodes trachyleberidídeos, os quais podem representar um ambiente nerítico interno a médio (0-100 m), enquanto que na amostra QUI 1 foram encontradas, juntamente com os foraminíferos bentônicos bolivinídeos, associações de foraminíferos planctônicos pertencentes aos gêneros Hedbergella e Whiteinella sugerindo um ambiente relativamente mais profundo, do nerítico externo ao batial superior (200-400 m) (Figura 7), estes dados estão de acordo com os de Hart $\&$ Koutsoukos (2015), que apresentaram uma reconstituição paleoecológica com associações de morfotipos planctônicos e bentônicos (incluindo os hedbergelídeos, bolivinídeos e textulariídeos) do Golfo do México e bacias das margens passivas do Oceano Atlântico.

\section{Considerações paleobiogeográficas}

De acordo com Leckie et al. (2002), durante o Cenomaniano-Coniaciano inferior, em regiões localizadas 


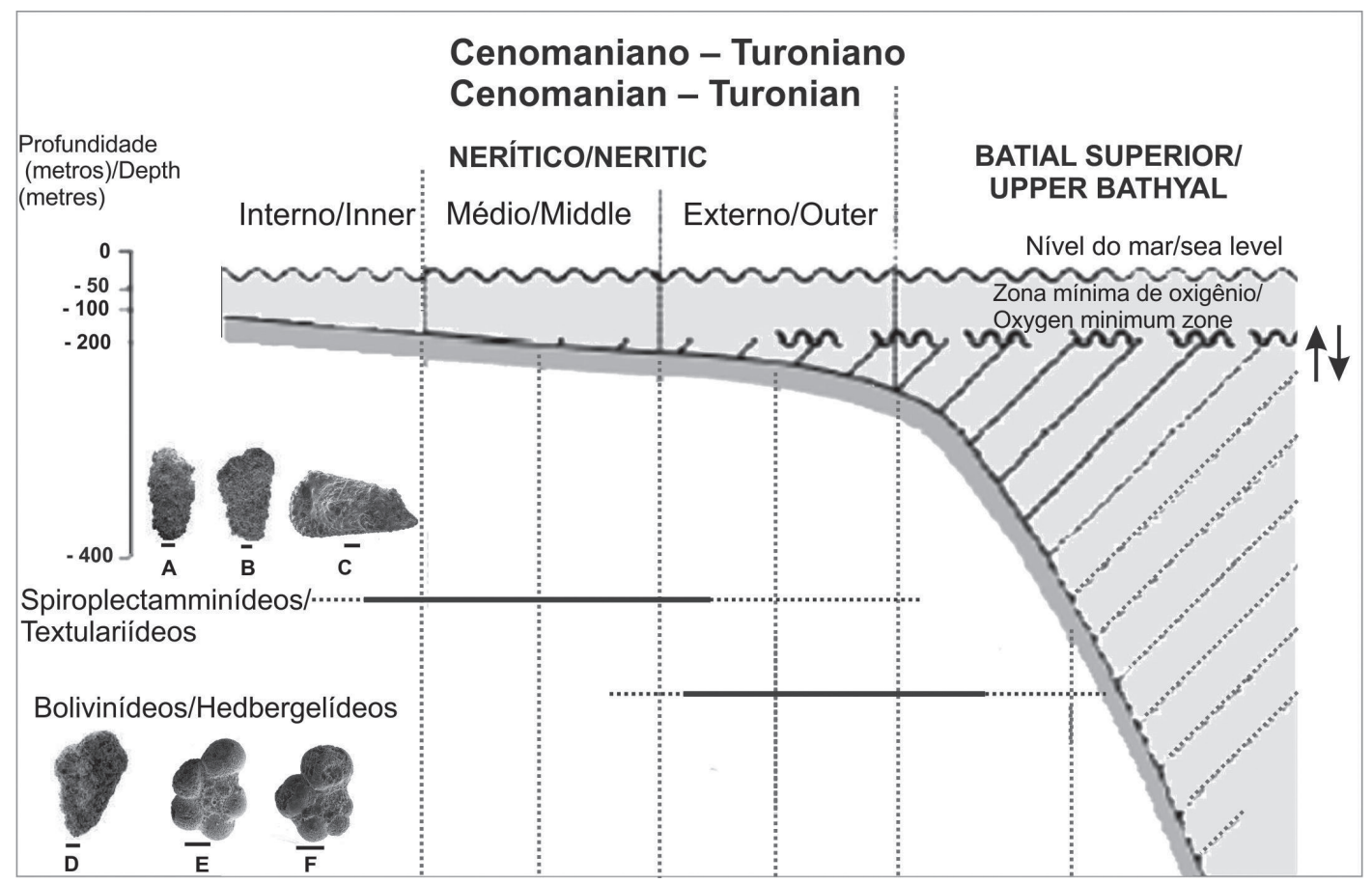

Figura 7. Reconstituição paleoecológica do afloramento Quiepe 1. Representando o ambiente nerítico interno a médio (QUI 2 e QUI3) os foraminíferos bentônicos: A, spiroplectamminídeo, B, textularí́deo, e o ostracode: C, trachyleberidídeo. Representando o ambiente nerítico externo ao batial superior o foraminífero bentônico: $\mathbf{D}$, bolivinídeo e os foraminíferos planctônicos hedbergelídeos: $\mathbf{E}$, Whiteinella aff. W. aprica e F, Hedbergella sp. 1 (Modificado de Hart \& Koutsoukos, 2015).

Figura 7. Paleoecological reconstruction of outcroup Quiepe 1. Representing the internal to middle neritic environment (QUI 2 e QUI 3) the benthic foraminifera: A, spiroplectamminid. B, textulariid, and the ostracod: $\mathbf{C}$. trachyleberidid. Representing the neritic environment outer to the upper batial, the benthic foraminifera: $\mathbf{D}$, bolivinid and the planktonic foraminifera hedbergellid: $\mathbf{E}$, Whiteinella aff. W. aprica and $\mathbf{F}$, Hedbergella sp. 1 (figure modified from Hart \& Koutsoukos, 2015).

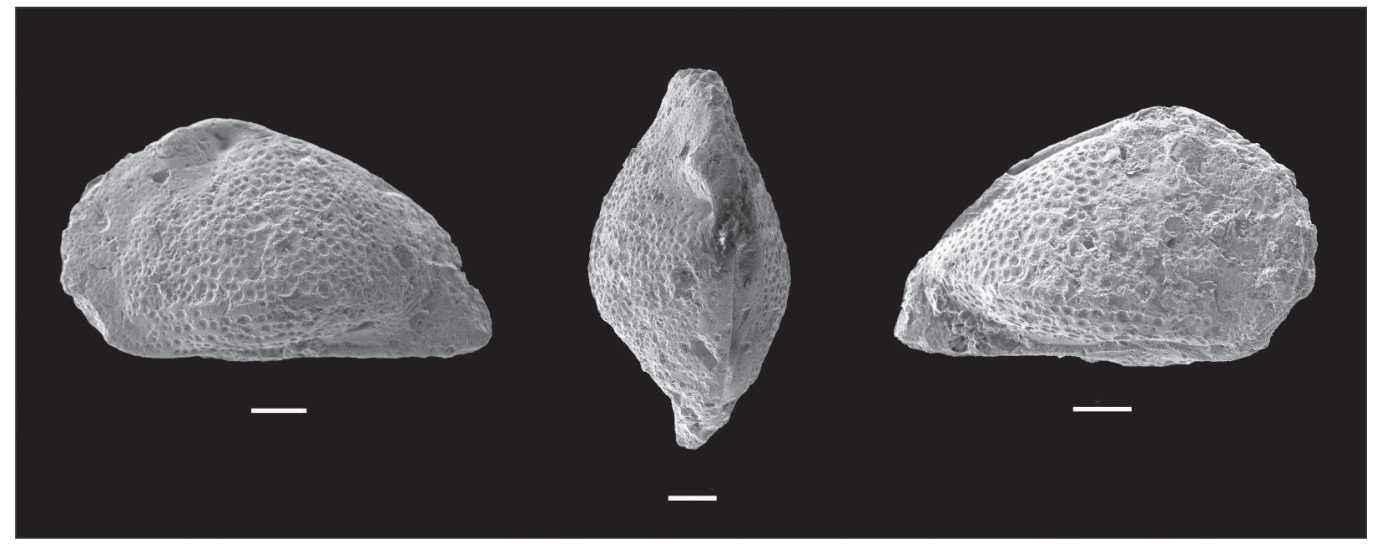

Figura 8. A-C, Sapucariella ex. gr. sapucariensis (Pukett et al., 2016). A, vista lateral da valva esquerda. B, vista lateral da valva direita. C vista dorsal. Recuperado nos afloramentos da Ilha de Quiepe (QUI 1), Bacia de Camamu, Bahia, Brasil. Escalas $=100 \mu \mathrm{m}$.

Figure 8. A-C, Sapucariella ex. gr. sapucariensis (Pukett et al., 2016). A, side view of the left valve. B, side view of the right valve. C, dorsal view. Recovered from outcrops of Quiepe Island (QUI 1), Camamu Basin, Bahia, Brazil. Scale bars $=100 \mu \mathrm{m}$.

em latitudes baixas do Atlântico Sul, como a Bacia de Sergipe e outras bacias do Nordeste do Brasil, condições de clima quente e de relativa regularidade predominavam e favoreciam o aumento das taxas de evaporação. Devido às restrições paleofisiográficas das bacias, a circulação de fundo era reduzida (Koutsoukos, 1989). Concomitantemente, as massas de água tornaram-se estratificadas (variação de salinidade), ocorrendo a depleção de oxigênio nas camadas de fundo. No Neocretáceo, em razão da predominância de condições paleoambientais aeróbicas e oligotróficas, novos nichos paleoecológicos foram gerados e a diversidade dos foraminíferos planctônicos e bentônicos se desenvolveu em paleocomunidades reconhecidas pela complexidade de sua estrutura trófica (Koutsoukos \& Hart, 1990). 


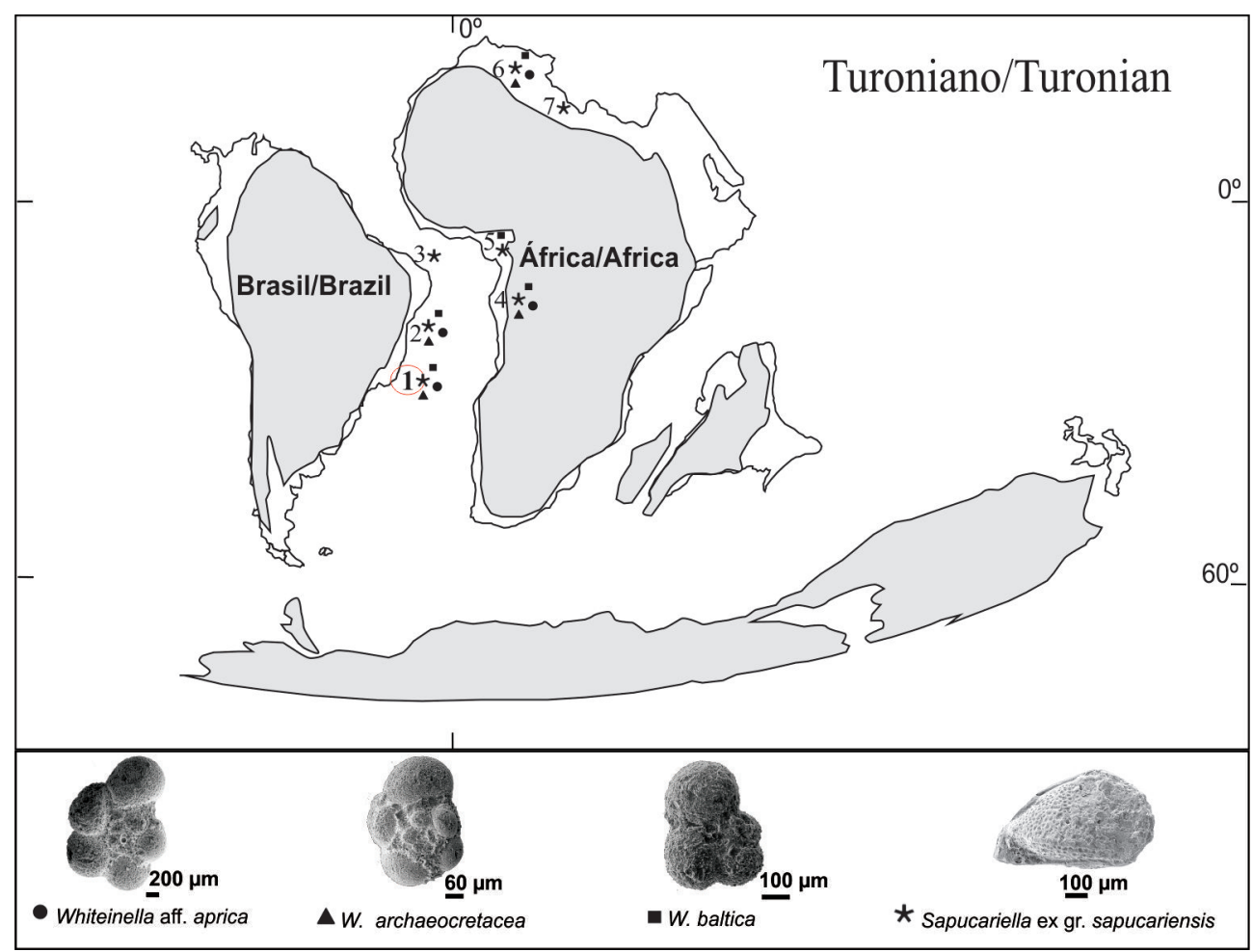

Figura 9. Distribuição paleobiogeográfica das espécies de foraminíferos planctônicos Whiteinella aff. $W$. aprica, $W$. archaeocretacea, $W$. baltica e do ostracode Sapucariella ex gr. sapucariensis no Turoniano (modificado de Smith et al., 1994). 1, Bacia de Camamu (este estudo; Mascarenhas et al., 2016). 2, Bacia de Sergipe (Krömmelbein, 1964; Neufville, 1973; Koutsoukos, 1989, 1992; Koutsoukos \& Bengtson, 1993; Viviers et al., 2000). 3, Bacia Potiguar (Viviers et al., 2000). 4, Bacia do Gabão (Grosdidier, 1979; Neufville, 1973, Koutsoukos, 1989, 1992; Koutsoukos \& Bengtson, 1993; Dupont, 1996). 5, Nigéria Oriental (Neufville, 1973; Igwe et al., 2015). 6, Marrocos (Andreu, 1996). 7, Tunísia (Bismuth et al., 1981).

Figure 9. Paleobiogeographic distribution of the planktonic foraminifera Whiteinella aff. W. aprica, W. archaeocretacea, W. baltica and the ostracod Sapucariella sapucariensis, in the Turonian (figure modified from Smith et al., 1994). 1, Camamu Basin (Mascarenhas et al., 2016). 2, Sergipe Basin (Krömmelbein, 1964; Neufville, 1973; Koutsoukos, 1989, 1992; Koutsoukos \& Bengtson, 1993; Viviers et al., 2000). 3, Potiguar Basin (Viviers et al., 2000). 4, Gabon Basin (Grosdidier, 1979; Koutsoukos, 1989, 1992; Koutsoukos \& Bengtson, 1993; Dupont, 1996). 5, Eastern Nigeria (Neufville, 1973; Igwe et al., 2015). 6, Morocco (Andreu, 1996). 7, Tunisia (Bismuth et al., 1981).

Os estudos com foraminíferos planctônicos e ostracodes realizados em bacias cretáceas no Nordeste do Brasil (Koutsoukos, 1989, 1992; Viviers et al., 2000) foram utilizados para caracterizar regiões paleobiogeográficas. Segundo Koutsoukos (1992), as condições paleoceanográficas que se desenvolveram no Cenomaniano, mesmo com algumas variações, perduraram até o Turoniano. Neste cenário, as associações de foraminíferos bentônicos eram dominadas por buliminídeos, bolivinídeos e textulariídeos. Já as associações de planctônicos eram predominantemente constituídas por globigerinelloidídeos, guembelitriídeos, heterohelicídeos e hedbergelídeos. Dentre esses últimos, foram observadas diversas espécies do gênero Whiteinella (W. aprica, $W$. archaeocretacea, $W$. baltica, $W$. brittonensis e $W$. paradubia). Os táxons Whiiteinella aff. W. aprica, W. archaeocretacea e $W$. baltica estão bem representados na seção turoniana da Ilha de Quiepe da Formação Algodões, mesmo apresentando-se como uma associação pouco diversificada. De acordo com Viviers et al. (2000) e Puckett et al. (2016), ostracodes da família Brachycytheridae e, especialmente, Sapucariella sapucariensis, mostraram, durante o Turoniano, uma expressiva distribuição paleogeográfica ao longo das bacias das margens atlânticas da América do Sul e da África (Figura 8). No Brasil, nas associações de ostracodes desses intervalos de idade turoniana, foram registradas as espécies de Sapucariella do grupo sapucariensis nas bacias de Sergipe, Potiguar e Camamu (Viviers et al., 2000; Puckett et al., 2016; Mascarenhas et al., 2016).

Puckett et al. (2016) realizaram estudos paleogeográficos onde descreveram seis gêneros para a família Brachycytheridae: Acuminobrachycythere, Brachycythere, Kaesleria (restrito para o Oriente Médio), Opimocythere (para o Paleógeno da América do Norte) e os gêneros Sapucariella e Tricostabrachycythere. Estes dois últimos considerados como limitados ao Supercontinente Gondwana. Inferências paleogeográficas relacionadas à origem e evolução desses gêneros foram discutidas pelos autores, os quais apresentaram modelos de mapas com reconstruções paleogeográficas do processo de separação da Pangea relacionadas a estes ostracodes marinhos cretáceos. No Cretáceo, os ostracodes pertencentes ao gênero Sapucariella tiveram sua ocorrência localizada na América do Sul e África e os pertencentes ao gênero Brachycythere foram restritos à América do Norte. 
Os registros dos foraminíferos planctônicos Whiteinella aff. W. aprica, W. archaeocretacea, $W$. baltica e do ostracode Sapucariella ex gr. sapucariensis (Figura 8), na exposição aflorante da Ilha de Quiepe, Formação Algodões, Bacia de Camamu, apresentam correlação com os trabalhos de Koutsoukos $(1989,1992)$ e Viviers et al. (2000), que também registraram Whiteinella aprica, $W$. archaeocretacea, W. baltica e Sapucariella sapucariensis nas seções estratigráficas do Cenomaniano-Turoniano da Bacia de Sergipe. A observação dessas espécies de foraminíferos e ostracodes sugere que as bacias de Sergipe e Camamu são correlacionáveis paleobiogeograficamente. As associações desses microfósseis possuem alta afinidade nas bacias do Nordeste do Brasil (Sergipe e Potiguar) e bacias da África e atestam semelhantes condições paleoceanográficas, em ambas margens do Atlântico que estavam em latitudes baixas durante o Turoniano (Grosdidier, 1979; Koutsoukos, 1989, 1992; Andreu, 1996; Dupont, 1996; Viviers et al., 2000; Puckett et al., 2016; Mascarenhas et al., 2016).

Leckie (1985) realizou estudos na Formação Greenhorn, Colorado (USA), onde identificou espécies de foraminíferos planctônicos do Cenomaniano-Turoniano, incluindo Whiteinella aprica; $W$. archaeocretacea e W. baltica. Dupont (1996) realizou um trabalho taxonômico e bioestratigráfico com as principais espécies de foraminíferos planctônicos da Bacia do Gabão, descrevendo dentre ouras as espécies de $W$. aprica, W. archaeocretacea, W. baltica (Cenomaniano superior-Turoniano médio). Igwe et al. (2015) também constataram foraminíferos planctônicos de idade turoniana em afloramentos da Nigéria. Os autores registraram associações de foraminíferos planctônicos constituídas pelos gêneros Hedbergella e Whiteinella. Dentre as espécies identificadas, encontra-se $W$. baltica, aqui registrada para os afloramentos da Ilha de Quiepe.

O mapa (Figura 9) mostra a distribuição paleobiogeográfica no Turoniano das espécies de foraminíferos planctônicos Whiteinella aprica, W. archaeocretacea, W. baltica e do ostracode Sapucariella sapucariensis, para as bacias de Camamu (este estudo); Sergipe (Krömmelbein, 1964; Neufville, 1973; Koutsoukos, 1989, 1992; Koutsoukos \& Bengtson, 1993; Viviers et al., 2000); Potiguar (Viviers et al., 2000); Gabão (Grosdidier, 1979; Koutsoukos, 1989, 1992; Dupont, 1996); Nigéria Oriental (Neufville, 1973; Igwe et al., 2015); Marrocos (Andreu, 1996) e Tunísia (Bismuth et al., 1981).

Os foraminíferos planctônicos (Witheinella aprica, $W$. archaeocretacea, $W$. baltica e Whiteinella sp. 1), associados ao ostracode $S$. ex gr. sapucariensis, contribuem com dados bioestratigráficos e paleobiogeográficos para o CenomanianoTuroniano das bacias marginais brasileiras. Além disso, a composição taxonômica e a distribuição bioestratigráfica desses foraminíferos planctônicos e bentônicos e ostracodes, do Turoniano da Ilha de Quiepe, demostraram a semelhança faunística e confirmaram afinidades palebiogeográficas das associações microfaunísticas da Formação Algodões na Bacia de Camamu e outras bacias coevas, particularmente com a Bacia de Sergipe.

\section{CONSIDERAÇÕES FINAIS}

Este trabalho apresenta o primeiro registro de foraminíferos planctônicos e bentônicos em afloramentos da Ilha de Quiepe (Membro Quiepe), Formação Algodões, Bacia de Camamu, Bahia, Brasil. Foram recuperados um total de 128 espécimes de foraminíferos, sendo 65 espécimes de foraminíferos planctônicos distribuídos entre os gêneros, Hedbergella (Hedbergella? sp. 1) e Whiteinella (W. aff. $W$. aprica, $W$. archaeocretacea, $W$. baltica e Whiteinella sp. 1) pertencentes à família Hedbergellidae; e 63 espécimes de foraminíferos bentônicos, pertencentes às famílias Bolivinidae? Spiroplectamminidae? e Textularinidae?.

A ocorrência e a amplitude estratigráfica das espécies de foraminíferos planctônicos Whiteinella aff. W. aprica, $W$. archaeocretacea $\mathrm{e} W$. baltica, identificadas nas amostras QUI 1, QUI 2 e QUI 3 do afloramento Quiepe 1, sugerem uma idade neocenomaniana-neoturoniana para a seção estudada da Formação Algodões, na Bacia Camamu. No arcabouço bioestratigráfico de referência (Encontro do Grupo de Trabalho de Microfósseis do Cretáceo Superior 2011 - Times Scale Creator, versão TSC 6.4), intervalos dessa idade se correspondem ao conjunto de biozonas Rotalipora cushmani, Whiteinella archaeocretacea, Helvetoglobotruncana helvetica e Marginotruncana schneegansi, que no presente trabalho não foram individualizadas ante a falta de registro das espécies diagnósticas (salvo $W$. archaeocretacea), numa associação planctônica pouco diversificada. Estes dados estão em parte de acordo com Andrade et al. (2006), que, com base em amonoides, inferiram idade eo-mesoturoniana para o afloramento da Ilha de Quiepe. A integração entre os dados bioestratigráficos fornecidos pelos foraminíferos planctônicos e os amonoides permite restringir ao Eomesoturoniano a idade do afloramento da Formação Algodões na Ilha de Quiepe.

Os estudos paleoecológicos demostraram que as associações de foraminíferos bentônicos registradas na Ilha de Quiepe são constituídas por bolivinídeos, spiroplectamminídeos e textulariídeos, associadas a ostracodes e amonoides, sugerindo um paleoambiente marinho formado por uma plataforma carbonática. Estas conclusões têm como base a predominância dos bolivinídeos na associação, e a ocorrência exclusiva de morfogrupos de hábito infaunais, que estão em consonância com o modelo de distribuição faunística proposto para o CenomanianoTuroniano da Bacia de Sergipe por Koutsoukos \& Hart (1990). As paleobatimetrias estimadas oscilaram entre o nerítico interno a profundo $(0-400 \mathrm{~m})$ para as amostras QUI 1, QUI 2 e QUI 3 do afloramento Quiepe 1.

A associação de foraminíferos planctônicos e ostracodes, registrada na Formação Algodões, representada pelas espécies Whiteinella aff. W. aprica, W. archaeocretacea, W. baltica e Sapucariella ex gr. sapucariensis apresentam afinidades paleobiogeográficas com associações descritas para as bacias brasileiras de Sergipe e Potiguar e do Gabão, Nigéria e Marrocos, na África. 


\section{AGRADECIMENTOS}

Ao Programa de Pós-Graduação em Geologia da Universidade Federal da Bahia (UFBA). Ao CNPq pela bolsa concedida à primeira autora. Aos professores da UFBA, O.R. Leite e S.S. de Moraes, pelas orientações e colaboração durante os trabalhos de campo. Ao técnico responsável pelo Laboratório de MEV-CENPES/PDEP/BPA, R.S. Martins da Costa, pelas fotomicrografias dos exemplares de ostracodes e foraminíferos.

\section{REFERÊNCIAS}

Andrade, E.J.; Leite, O.R. \& Souza-Lima, W. 2006. Macrofauna do Membro Quiepe, Bacia de Camamu, Bahia, Brasil. Paleontologia em Destaque, 57:38-39.

Andrade, E.J.; Seeling, J.; Bengtson, P. \& Souza-Lima, W. 2004. The bivalve Neithea from the Cretaceous of Brazil. Journal of South American Earth Sciences, 17:25-38. doi:10.1016/j. jsames.2004.05.006

Andreu, B. 1996. Nouvelles espèces d'ostracodes du Turonien supérieur (?) e Coniacien (?) e Santonien de la région de Boulmane, Moyen Atlas, Maroc. Systématique, biostratigraphie et paléoécologie, paléobiogéographie des associations. Bulletin des Centres de Recherche Exploration-Production Elf-Aquitaine Mémoire, 16:483-509.

Babinot, J.F. 1995. Patterns of variability in ostracode species and communities from the late Cretaceous carbonate platforms: a report for ecozonal modelling and the study of ambient conditions. Palaeogeography, Palaeoclimatology, Palaeoecology, 119:93106. doi:10.1016/0031-0182(95)00062-3

Bengtson, P. 1983. The Cenomanian-Coniacian of the Sergipe Basin, Brazil. Fossils and Strata, 12:1-78.

Bismuth, H.; Boltenhagen, C.; Donze, P.; Le Fevre, J. \& SaintMarc, P. 1981. Le Cretace Moyen et Superieur de Djebel Semmama (Tunisie du Centre-Nord): Microstratigraphie et evolution sedimentologique. Bulletin des Centres de Recherches Exploration-Production Elf-Aquitaine Mémoire, 5:193-267.

Bolli, H.M.; Beckmann, J.P. \& Saunders, J.B. 1994. Benthic foraminiferal biostratigraphy of the South Caribbean Region. Cambridge, University Press, 408 p. doi:10.1017/ CBO9780511564406

Caixeta, M.J.; Milhomem, P.S.; Whitzke, R.E; Dupuy, I.S.S. \& Contijo, G.A. 2007. Bacia de Camamu. Boletim de Geociências da Petrobras, 15:455-461.

Caron, K. 1978. Cretaceous planktonic foraminifers from DSDP Leg 40, Southeastern Atlantic Ocean. Initial Reports of the Deep Sea Drilling Project, 40:651-678. doi:10.2973/dsdp. proc. 40.114 .1978

Caron, K. 1985. Cretaceous planktic foraminifera. In: H.M. Bolli; J.B. Saunders \& K. Perch-Nielsen (eds.) Plankton Stratigraphy, Cambridge University Press, p. 17-86.

Cavalier-Smith, T. 2002. The phagotrophic origin of eukaryotes and phylogenetic classification of Protozoa. International Journal of Systematic and Evolutionary Microbiology, 52:297-354. doi:10.1099/ijs.0.02058-0

D'orbigny, A. 1826. Tableau mkthodique de la classe des Cephalopodes. Annales des Sciences Naturelles, 7:245-314.

Douglas, R.G. \& Rankin, C. 1969. Cretaceous planktonic foraminifera from Bornholm and their zoogeographic significance. Lethaia, 2:185-217. doi:10.1111/j.1502-3931.1969.tb01848.x
Dupont, G. 1996. Principaux foraminifères planctiques du Crétacé gabonais (Aptien à Campanien inférieur). In: S. Jardiné; I. de Klasz \& J.-P.. Debenay (eds.). Géologie de l'Afrique et de l'Atlantique Sud, Angers, Université d'Angers, $p$.83-121.

Eicher, D.L. \& Worstell, P. 1970. Cenomanian and Turonian foraminifera from the Great Plains, United States. Micropaleontology, 16:269-324.

Fauth, G. 2002. Paleobiogeography of the Upper Cretaceous to Lower Tertiary marine ostracods from the Atlantic Ocean. Revista Brasileira de Paleontologia, 4:65-71.

Gebhardt, H. 1997. Cenomanian to Turonian foraminifera from Ashaka (NE Nigeria): Quantitative analysis and palaeoenvironmental interpretation. Cretaceous Research, 18:17-36. doi:10.1006/cres.1996.0047

Gradstein, F.M.; Ogg, J.G.; Schmitz, M.D. \& Ogg, G.M. 2012. The Geologic Time Scale. $1^{\text {a }}$ ed. Oxford, Elsevier, 1144 p.

Grosdidier, E. 1979. Principaux ostracodes marins de l'intervalle Aptien-Turonien du Gabon (Afrique Occidentale). Bulletin des Centres de Recherches Exploration-Production Elf-Aquitaine Mémoire, 3:1-35.

Hart, M.B. \& Koutsoukos, E.A.M. 2015. Paleoecology of Cretaceous foraminifera: examples from the Atlantic Ocean and Gulf of Mexico region. Gulf Coast Association of Geological Societies Transactions, 65:175-199.

Igwe, E.O.; Okuro, A.U. \& Obari, A.I. 2015. Foraminiferal biostratigraphic analysis of the late Cenomanian-Turonian EzeAku Shale in the Afikpo Synclinorium, Lower Benue Trough, Nigeria. American Journal of Science and Technology, 2:274-282.

Ingle Junior, J.C. 1980. Cenozoic paleobathymetry and depositional history of selected sequences within the Southern California continental borderland. In: W.V. Sliter (ed.) Studies in marine micropaleontology and paleoecology: a memorial volume to Orville L. Bandy, Houston, Cushman Foundation for Foraminiferal Research, p. 163-195 (Special Publication 19).

Koutsoukos, E.A.M. 1989. Mid-to Late Cretaceous microbiostratigraphy, palaeoecology and palaeogeography of the Sergipe Basin, Northeastern Brazil. Polytechnic South West, Tese de doutorado, $886 \mathrm{p}$.

Koutsoukos, E.A.M. 1992. Late Aptian to Maastrichtian foraminiferal biogeography and palaeoceanography of the Sergipe Basin, Brazil. Palaeogeography, Palaeoclimatology, Palaeoecology, 92:295-324. doi:10.1016/0031-0182(92)90089-N

Koutsoukos, E.A.M. \& Bengtson, P. 1993. Towards an integrated biostratigraphy of the Aptian-Maastrichtian of the Sergipe Basin, Brazil. Documents du Laboratoire de Géologie de Lyon, 125:241-262.

Koutsoukos, E.A.M. \& Hart, M.B. 1990. Cretaceous foraminiferal morphogroup distribution patterns, palaeocommunities and trophic structures: a case study from the Sergipe Basin, Brazil. Earth and Environmental Science Transactions of The Royal Society of Edinburgh, 81:221-246. doi:10.1017/ S0263593300005253

Krömmelbein, K. 1964. Ostracoden aus der marinen "Küsten-Kreide" Brasiliens. 1: Brachycythere (Brachycythere) sapucariensis n. $\mathrm{sp}$. aus dem Turonium. Senckenbergiana lethaia, 45:489-495.

Leckie, R.M. 1985. Foraminifera of the Cenomanian-Turonian boundary interval, Greenhorn Formation, Rock Canyon anticline, Pueblo, Colorado. In: L.M. Pratt; E.G. Kauffman \& F. Zelt (eds.) Fine-Grained Deposits and Biofacies of the Cretaceous Western Interior Seaway: Evidence of Cyclic Sedimentary Processes, Society of Economic Paleontologists and Mineralogists, p. 139-149. doi:10.2110/sepmfg.04.139 
Leckie, R.M.; Bralower, T.J. \& Cashman, R. 2002. Oceanic anoxic events and plankton evolution: Biotic response to tectonic forcing during the mid-Cretaceous. Paleoceanography, 17:1-29. doi:10.1029/2001PA000623

Loeblich Jr., A.R. \& Tappan, H.N. 1961. Cretaceous planktonic foraminifera; Part 1, Cenomanian. Micropaleontology, 7:257-304.

Loeblich Jr., A.R. \& Tappan, H.N. 1988. Foraminiferal Genera and Their Classification. New York, Van Nostrand Reinhold Company, $970 \mathrm{p}$.

Manso, L.C.C. 2003. Paleoecologia, paleogeografia e sistemática dos Equinoides Aptiano-Albiano (Cretáceo do Brasil). Programa de Pós-Graduação em Geologia, Universidade Federal da Bahia, Tese de doutorado, $196 \mathrm{p}$.

Manso, L.C.C. \& Souza-Lima, W. 2007. Holosalenia bahiensis, um novo equinoide (Echinodermata) do Albiano da bacia de Camamu, Brasil. Revista Brasileira de Paleontologia, 10:27-34.

Mascarenhas, G.B.C.; Piovesan, E.K. \& Machado, A.J. 2016. Ostracodes da Ilha de Quiepe (Turoniano) da Bacia de Camamu, Bahia, Brasil: taxonomia e interferências paleobiogeográficas. Revista Estudos Geológicos, 26:108-128. doi:10.18190/19808208/estudosgeologicos.v26n2p108-128

Murray, J. 2006. Ecology and Applications of Benthic Foraminifera. $1^{\mathrm{a}}$ ed. Cambridge, Cambridge University Press, 426 p.

Netto, A.S.T.; Filho, J.R.W. \& Feijó, F.J. 1994. Bacias de Jacuípe, Camamu e Almada. Boletim de Geociências da Petrobras, 8:173-184.

Neufville, M.H. 1973. Upper Cretaceous-Paleogene Ostracoda from the South Atlantic. Publications from the Paleontological Institution of the University of Uppsala, 1:1-193.

Noguti, I. \& Santos, J.F. 1972. Zoneamento preliminar por foraminíferos planctônicos do Aptiano ao Mioceno na plataforma continental do Brasil. Boletim Técnico da Petrobras, 15:265-283.

Piovesan, E.K.; Bergue, C.T. \& Fauth, G. 2009. Cretaceous ostracodes from Pará-Maranhão Basin, Brasil: taxonomy and preliminary paleoecological and paleobiogeographical inferences. Revue de Paléobiologie, 28:437-456.

Piovesan, E.K.; Cabral, M.C.; Colin, J.-P.; Fauth, G. \& Bergue, C.T. 2014a. Ostracodes from the Upper Cretaceous of the Potiguar Basin, Northeastern Brazil: taxonomy, paleoecology and paleobiogeography, Part 1: Turonian. Carnets de Géologie [Notebooks on Geology], 14:211-252. doi :10.4267/2042/54003

Piovesan, E.K.; Cabral, M.C.; Colin, J.-P.; Fauth, G. \& Bergue, C.T. 2014b. Ostracodes from the Upper Cretaceous of the Potiguar Basin, Northeastern Brazil: taxonomy, paleoecology and paleobiogeography, Part 2: Santonian-Campanian. Carnets de Géologie [Notebooks on Geology], 14:315-351. doi:10.4267/2042/54151

Premoli-Silva, I. \& Verga, D. 2004. Pratical Manual of Cretaceous Planktonic Foraminifera, Couse 3. In: D. Verga \& R. Rettori (eds.) International School on Planktonic Foraminifera, Universities of Perugia and Milano, $283 \mathrm{p}$.

Puckett, T.M.; Andreu, B. \& Colin, J.-P. 2016. The evolution of the Brachycytheride Ostracoda in the contexto of the breakup of Pangea. Revue de Micropaléontologie, 59:97-167. doi:10.1016/j.revmic.2016.04.001

Robaszynski, F. \& Caron, M. 1979. Atlas de Foraminiféres planctoniques du Crétacé moyen (mer boreale et Téthys). Cahiers de Micropaléontologie, 1:1-185, 2:1-181.

Schaller, H. 1969. Revisão estratégica da bacia de Sergipe/Alagoas. Boletim Técnico da Petrobrás, 12: 21-86.

Silva, C.P. 2007. Estudo sobre foraminiferos e radiolários do Cretáceo, Bacia Pará-Maranhão, margem equatorial brasileira. Programa de Pós-Graduação em Geociências, Universidade Federal do Rio Grande do Sul, Dissertação de mestrado, 151 p.

Silva, C.P. 2011. Bioestratigrafia e paleoecologia de foraminiferos da Bacia de Barreirinhas, Cretáceo, margem equatorial brasileira. Programa de Pós-Graduação em Geociências, Universidade Federal do Rio Grande do Sul, Tese de doutorado, 132 p.

Smith, A.G.; Smith, D.G. \& Funnel, B.M. 1994. Atlas of Mesozoic and Cenozoic coastlines. Cambridge, Cambrige University Press, 99 p.

Souza-Lima, W. 2003a. Nova ocorrência de amonoide no Albiano da bacia de Camamu, Bahia, Brasil. In: REUNIÃO ANUAL REGIONAL DA SOCIEDADE BRASILEIRA DE PALEONTOLOGIA, 2002. Resumos, Aracaju, p. 7.

Souza-Lima, W. 2003b. Registro de corais no Albiano da bacia de Camamu, Bahia, Brasil. In: REUNIÃO ANUAL REGIONAL DA SOCIEDADE BRASILEIRA DE PALEONTOLOGIA, 2002. Resumos, Aracaju, p. 11.

Souza-Lima, W.; Andrade, E.J. \& Manso, C.L.C. 2001a. Novos estudos paleontológicos na Formação Algodões, Albiano da bacia de Camamu, Bahia, Brasil. In: CONGRESSO BRASILEIRO DE PALEONTOLOGIA, 17, 2001. Boletim de Resumos, Rio Branco, SBP, p. 57.

Souza-Lima, W.; Andrade, E.J. \& Manso, C.L.C. 2001 b. Considerações geológicas e paleontológicas sobre a seção marinha aflorante da bacia de Camamu, Bahia, Brasil. Revista Brasileira de Paleontologia, 2:155-156.

Viviers, M.C.; Koutsoukos, E.A.M.; Silva-Telles Jr., A.C. \& Bengtson, P. 2000. Stratigraphy and biogeographic affinities of the late Aptian-Campanian ostracods of the Potiguar and Sergipe basins in Northeastern Brazil. Cretaceous Research, 21:407-455. doi:10.1006/cres.2000.0205

Wanderley, M.D. 2004. Técnicas de preparações de microfósseis. In: I.S. Carvalho (ed.) Paleontologia, Interciência, p. 17-25.

Wonders, A.A. 1980. Middle and late Cretaceous planktonic Foraminifera of the western Mediterranean area. Utrecht Micropaleontology Bulletin, 24:1-158.

Received in May, 2016; accepted in April, 2017. 